\title{
Examining the provenance of branched GDGTs in the Tagus River drainage basin and its outflow into the Atlantic Ocean over the Holocene to determine their usefulness for paleoclimate applications
}

\author{
Lisa Warden ${ }^{1}$, Jung-Hyun Kim ${ }^{1, \mathrm{a}}$, Claudia Zell ${ }^{1, \mathrm{~b}}$, Geert-Jan Vis ${ }^{2}$, Henko de Stigter ${ }^{3}$, Jérôme Bonnin ${ }^{4}$, and \\ Jaap S. Sinninghe Damsté ${ }^{1,5}$ \\ ${ }^{1}$ NIOZ Netherlands Institute for Sea Research, Department of Marine Microbiology and Biogeochemistry, \\ and Utrecht University, P.O. Box 59, 1790 AB Den Burg, the Netherlands \\ ${ }^{2}$ TNO, Geological Survey of the Netherlands, P.O. Box 80015, 3508 TA Utrecht, the Netherlands \\ ${ }^{3}$ NIOZ Netherlands Institute for Sea Research, Department of Ocean Systems Sciences, and Utrecht University, \\ P.O. Box 59, 1790 AB Den Burg, the Netherlands \\ ${ }^{4}$ UMR-EPOC5805 CNRS, Université de Bordeaux, Allée Geoffroy St Hilaire, 33615 Pessac, France \\ ${ }^{5}$ Utrecht University, Faculty of Geosciences, Department of Earth Sciences, P.O. Box 80021, 3508 TA Utrecht, \\ the Netherlands \\ ${ }^{a}$ present address: Korea Polar Research Institute, 26 Songdomirae-ro, Yeonsu-gu, Incheon 21990, South Korea \\ ${ }^{b}$ present address: Pierre and Marie Curie University, Paris 6, METIS, UMR7619, France \\ Correspondence to: Lisa Warden (lisa.warden@nioz.nl)
}

Received: 18 April 2016 - Published in Biogeosciences Discuss.: 30 May 2016

Revised: 13 September 2016 - Accepted: 17 September 2016 - Published: 17 October 2016

\begin{abstract}
The distributions of branched glycerol dialkyl glycerol tetraethers (brGDGTs), which are transported from the soils where they are predominantly produced to marine sediments via rivers, have been applied in reconstructing mean annual air temperature (MAT) and $\mathrm{pH}$ of soils. However, paleoclimate reconstructions using sedimentary brGDGTs have proven difficult in arid regions, including the Iberian Peninsula. Recently, six novel 6-methyl brGDGTs have been described using new analytical methods (in addition to the nine 5-methyl brGDGTs previously used for climate reconstructions), and so new $\mathrm{pH}$ and MAT calibrations have been developed that were shown to improve the accuracy of reconstructions in a set of global soil samples, especially in arid regions. Because of this we decided to apply the new method to separate the 5- and 6-methyl isomers along with the novel calibrations to a sample set from the Iberian Peninsula to determine whether it improves paleoclimate reconstructions in this area. This set includes samples that run in a transect from source to sink along the Tagus River and out to the deep ocean off the Portuguese margin spanning the last 6000 years. We found that although $\mathrm{pH}$ reconstructions
\end{abstract}

in the soils were improved using the new calibration, MAT reconstructions were not much better even with the separation of the 5- and 6-methyl brGDGTs. This confirmed the conclusion of previous studies that the amount of aquatically produced brGDGTs is overwhelming the soil-derived ones in marine sediments and complicating MAT reconstructions in the region. Additionally, the new separation revealed a strong and until now unseen relationship between the new degree of cyclization (DC') of the brGDGTs and MAT that could be making temperature reconstructions in this and other arid regions difficult.

\section{Introduction}

Understanding past climate variability is important for predicting future climate change as well as how ecosystems, organisms, and human society could be affected. The validation of climate proxies is imperative for the correct interpretation of climate archives and therefore also for the climate models building on these past climate data. Terrestrial environments 
play an important role in global climate; however, continental climate reconstructions are hindered by the lack of continental temperature proxies. In the future, changes in terrestrial climate are likely to have a large impact on human society just as they had in the past (e.g., Haug et al., 2003). Availability of trustworthy temperature data from the terrestrial environment will be essential for the development of reliable climate models.

The distribution of branched glycerol dialkyl glycerol tetraethers (brGDGTs, Fig. S1 in the Supplement), a group of membrane-spanning lipids that occur in heterotrophic bacteria (Pancost and Sinninghe Damsté, 2003; Weijers et al., 2010) pervasive in peat (Weijers et al., 2006) and worldwide in soils has proven useful as a tool to obtain highresolution, continental temperature reconstructions (Weijers et al., 2007a; Schouten et al., 2008; Bendle et al., 2010). Branched GDGTs are biosynthesized by bacteria (Sinninghe Damsté et al., 2011, 2014) living in soils and the distribution of brGDGTs in soils is affected by growth temperature and $\mathrm{pH}$ (Weijers et al., 2006). More specifically, the degree of methylation of the brGDGTs (expressed as methylation of branched tetraethers index, MBT; see Table S1 in the Supplement for a detailed explanation of all GDGT indices used in this study) relates to mean annual air temperature (MAT), and to a lesser extent soil $\mathrm{pH}$, whereas the degree of cyclization (DC) of the brGDGTs (also expressed as the cyclization of branched tetraethers index, CBT) correlates solely with soil pH (Weijers et al., 2007a). MBT has recently been amended to become MBT' by eliminating the brGDGTs that rarely occur in soils (Peterse et al., 2012). These observations led to the development of a continental paleoclimate proxy based on the distribution of brGDGTs that has been applied in paleosoils (Peterse et al., 2009; Weijers et al., 2007a, 2011). Branched GDGTs that are produced in soils are washed by runoff into streams and rivers, where they are transported to and deposited in river and coastal marine sediments that are under the influence of major river systems. In this way, brGDGTs have been used as recorders of the continental paleoclimate (Weijers et al., 2007b; Bendle et al., 2010; Hren et al., 2010; Keating-Bitonti et al., 2011).

Complications using brGDGTs as a proxy for MAT have arisen in some settings. In marine sediments receiving a low input of soil organic matter (OM), it was found that the distribution of brGDGTs and the reconstructed temperatures were quite different from that observed in regional soils (Peterse et al., 2009). Peterse et al. (2012) found in arid regions that temperature is no longer an important control on the distribution of brGDGTs and therefore MAT reconstructions in these areas should be interpreted with care. In the Iberian Peninsula, Menges et al. (2014) found that MBT' was not correlated with MAT but instead with the aridity index (AI), a parameter for water availability in soils, and mean annual precipitation (MAP). In drainage basins with varying soil sources that had different MATs (i.e., mountainous vs. lowland), it was found that the provenance of the soil matter must be consid- ered when interpreting MAT reconstructions (Bendle et al., 2010). In situ production of brGDGTs can occur within the river systems (Yang et al., 2012; Zell et al., 2013; De Jonge et al., 2014b) and cause brGDGT distributions and MAT reconstructions that differ from those in the soils of the source area. These complications make it vital to investigate how varying environmental conditions, the transport of these terrestrially derived fossilized lipids, and in situ production affect the implementation of brGDGTs for paleoclimate reconstructions.

Recently, a set of six new brGDGT isomers that differ in the position of the methyl groups were identified and described (De Jonge et al., 2013). The relative abundance of these novel, 6-methyl brGDGTs is strongly dependent on $\mathrm{pH}$, and so by excluding them from the MBT' index (newly defined as MBT' ${ }_{5 \mathrm{ME}}$ ) the correlation with MAT is improved (De Jonge et al., 2014a). The CBT index was also redefined in this study, as CBT', to include all of the $\mathrm{pH}$-dependent 6-methyl brGDGTs and consequently yielded a higher correlation with soil pH as a result (De Jonge et al., 2014a). De Jonge et al. (2014a) also developed, based on a dataset of globally distributed soils, a new $\mathrm{pH}$ calibration taking into account the new CBT' as well as new MAT calibrations, defined as $\mathrm{MAT}_{\mathrm{mr}}$ and $\mathrm{MAT}_{\mathrm{mrs}}$. In a global soil set they were shown to improve the accuracy of reconstructions, especially in arid regions. These indices and calibrations were applied in a coastal sediment core in the northern Kara Sea off Siberia in a study emphasizing the importance of examining the provenance of brGDGTs when using these lipids for paleoclimate reconstructions (De Jonge et al., 2015).

A comprehensive study has been previously performed on the present-day transport of brGDGTs in the Tagus River basin from source to sink (Zell et al., 2014). The results from this study demonstrated that the distribution of brGDGTs in the riverine suspended particulate matter (SPM) did not reflect that of the soils, implying that, due to the aquatic production in river and marine environments, the use of brGDGTs for paleoclimate reconstructions in the region would be complicated (Zell et al., 2014, 2015). Here we examine whether the assessment of the provenance of brGDGTs in the Tagus River basin can be improved by the application of analytical methods allowing for the separation of the 5- and 6-methyl brGDGTs (De Jonge et al., 2013). In addition, we examine whether the provenance of brGDGTs changed over the Holocene and whether the distribution of brGDGTs in the past reflected continental sources and thus past temperature and $\mathrm{pH}$ of the soils in the drainage basin of the river. To this end we compare the down-core brGDGT distributions in Holocene sediments retrieved from four locations along a transect in the Tagus River basin - which includes the river floodplain (Tagus River Floodplain core), the offshore mudbelt (Tagus Mudbelt core), and marine sediments from the canyons (Lisbon Canyon Head core and Lower Sétubal Canyon core; Fig. 1) - and compare them to brGDGT distributions of soil and river SPM from the Tagus River watershed. This allows insight into the potential and 


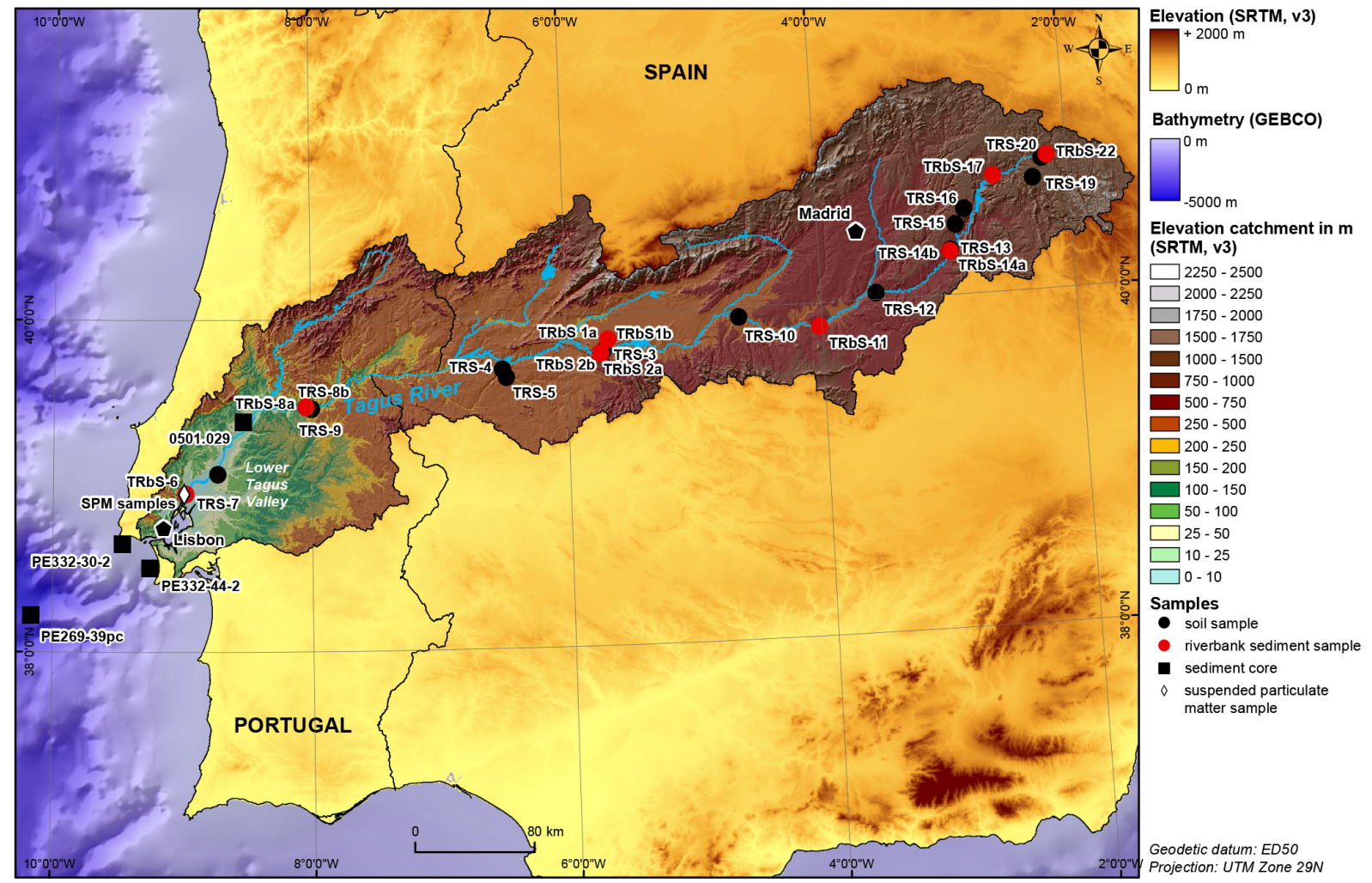

Figure 1. The location of the study area on the Iberian Peninsula with the stations where the four sediment cores were sampled (indicated by black squares) along a transect from the Tagus River to off the Portuguese continental margin, as well as the river SPM sampling site (indicated by a white diamond), riverbank sediment sampling sites (indicated by red circles), and soil sampling sites (indicated by black circles). The river SPM, riverbank sediments, and soil samples were all collected in a previous study. Digital elevation data are from Jarvis et al. (2006) and bathymetry from IOC-IHO-BODC (2003).

limitations of using the novel $\mathrm{MAT}_{\mathrm{mrs}} / \mathrm{CBT}$ ' proxies for climate reconstruction in this region and in river systems in general.

\subsection{Study area}

The Tagus River drains the central part of the Spanish Plateau with an E-W orientation (Benito et al., 2003). The waters originate at an elevation of about $1600 \mathrm{~m}$ altitude in eastern Spain at the Iberian Range and the mouth of the river feeds into the Atlantic Ocean near Lisbon (Vis and Kasse, 2009). At $1200 \mathrm{~km}$ long, the Tagus River is the longest river of the Iberian Peninsula and it occupies $82 \times 10^{3} \mathrm{~km}^{2}$, making it the third largest in catchment area (Benito et al., 2003). The Tagus Basin is surrounded by mountains on three sides with the Iberian Range to the east, the Central Range to the north, and the Toledo Mountains to the south. Present-day mean discharge at the Tagus River mouth is $400 \mathrm{~m}^{3} \mathrm{~s}^{-1}$ (Vale and Catarino, 1996; Vaz et al., 2011) and the largest contribution of draining tributaries comes from the Central Range in the north (Benito et al., 2003). The Tagus River is characterized by extreme seasonal and annual variability, including periods of flooding with 30 times the mean discharge and an annual discharge cycle characterized by two peaks in the winter (December and then again in February to March) and a discharge minimum in the summer (August; Benito et al., 2003). Since the 1940s dams have been built along the expanse of the Tagus River for water supply, hydropower, and flood prevention (Dias et al., 2002), which have likely impacted the transport of brGDGTs in the Tagus River system since their construction.

Where the Tagus River debouches into the Atlantic Ocean, the narrow continental shelf and steep continental slope are deeply incised by the Lisbon-Setúbal canyon system. The head of the Lisbon branch of that canyon system is located $13 \mathrm{~km}$ offshore from the Tagus River mouth at $120 \mathrm{~m}$ water depth. From that point, the canyon descends over a length of $165 \mathrm{~km}$ until it opens out onto the Tagus Abyssal Plain at $4860 \mathrm{~m}$ (Lastras et al., 2009). Even though the shelf is very narrow, sparse amounts of continental organic matter and clastic sediment reach the deep ocean in this region (Jounneau et al., 1998; de Stigter et al., 2011; Vis et al., 2016). This is because the Lisbon-Setúbal canyon is not a very dynamic system and has a weak down-canyon transport of sediments (Jouanneau et al., 1998; Jesus et al., 2010; de Stigter et al., 
2011). A part of the continental shelf in this region is covered by mud deposits, which originate predominantly from the Tagus estuary (Jouanneau et al., 1998). According to this same study, the mouth of the much smaller Sado River is located further to the southeast and contributes only a relatively minor sediments volume to the shelf mud deposits.

Generally, the climate of the Tagus River basin is characterized by seasonal variability and is considered continental Mediterranean (Le Pera and Arribas, 2004). Summers in the Tagus region are hot and dry and the winters are relatively mild and wet (Benito et al., 2003). During the summers, the climate regime in the Tagus Basin is controlled by the Azores High and in the winter by the westerlies (Benito et al., 2003). The MAT in the interior regions of the Tagus River basin varies from 7.5 to $12.5^{\circ} \mathrm{C}$ from the highlands to the lowlands of the inner basin, respectively, and can increase up to $16^{\circ} \mathrm{C}$ along the Atlantic coast (Le Pera and Arribas, 2004). The mean annual precipitation in the lowlands of the inner basin is mostly below $500 \mathrm{~mm}$, making it an arid region; however, some of the highest altitudes of the mountainous areas have a larger mean annual precipitation ranging from 750 to $1200 \mathrm{~mm}$ (Le Pera and Arribas, 2004).

The Iberian Peninsula is located between two major pressure systems, the Azores High and the Iceland Low, which make up the North Atlantic Oscillation (NAO). This climate phenomenon is caused by the varying pressure gradient in the North Atlantic and greatly influences climate conditions all over Europe (Hurrell, 1995; Hurrell and VanLoon, 1997). Because of the Iberian Peninsula's advantageous position for studying the shifting NAO, the climate in this region has been intensively investigated (Zorita et al., 1992; Rodó et al., 1997; Trigo et al., 2004). Many of these studies are from an oceanic perspective, obtaining sea surface temperatures from marine sediments using the alkenone unsaturation indices (Abrantes et al., 2005, 2009; Rodrigues et al., 2009), coccolithophore assemblages (Cachao and Moita, 2000; Palumbo et al., 2013), and stable isotopic oxygen composition of foraminifera (Lebreiro et al., 2006; Bartels-Jónsdóttir et al., 2006, 2009). The terrestrial climate has been examined using continental paleoarchives such as speleothems (MunozGarcia et al., 2007; Martin-Chivelet et al., 2011; Stoll et al., 2013), tree rings (Andreu et al., 2007; Linan et al., 2012), and pollen (Huntley and Prentice, 1988; Lebreiro et al., 2006; Davis et al., 2003; Fletcher et al., 2007; Corella et al., 2013). The integrated continental and marine approach can give complimentary information to past climate in a region, and by using the same proxy on the continent, in the ocean, and at the ocean-continent interface we would perhaps obtain a clearer picture of continental climate processes in an area than by using separate studies or a multi-proxy approach.

\section{Material and methods}

\subsection{Sample collection}

Soil samples, riverbank sediment samples, and river SPM from the Tagus River basin (Fig. 1b) were collected previously (Zell et al., 2014). These samples were complemented with four long sediment cores collected along a transect running from the Tagus River to the lower continental slope (Fig. 1). The Tagus River Floodplain core (0501.029) was collected in a low-energy backswamp of the present-day floodplain of the river at $\sim 4 \mathrm{~km}$ west of the Tagus channel (Table 1). The sediment was collected using an Edelman hand auger for sediment above the groundwater table and a gauge for sediment below the groundwater table (Vis et al., 2008). The sediments were wrapped in the field for laboratory analyses. The other three cores were collected using a piston corer, during campaigns in May 2007 and March 2011 with RV Pelagia conducted by the NIOZ Royal Netherlands Institute for Sea Research. The coring site for the Tagus Mudbelt core (64PE332-30-2) was to the west of the Tagus Estuary mouth, for the Lisbon Canyon Head core (64PE33244-2) it was to the east of the Tagus Estuary mouth, and for the Lower Setúbal Canyon core (64PE269-39) it was on the crest of the northern levee of the lower Setúbal Canyon (Table 1). A detailed description of the cores used in this study is given in the Supplement.

\subsection{Age models}

Accelerated mass spectrometry (AMS) ${ }^{14} \mathrm{C}$ measurements of the three marine sediment cores were carried out at the Beta Analytic laboratory (USA) on benthic or planktonic forams, gastropods, or shells fragments (Table 2). As for the Tagus River Floodplain core, the radiocarbon dating material was performed for a previous study and consisted of mostly terrestrial botanical macrofossils, but other bulk material was used as well (Vis et al., 2008). In order to establish consistent chronologies for the four sediment cores, all the AMS dates were calibrated into calendar ages using CALIB 7.0, available at http://radiocarbon.pa.qub.ac.uk/calib (Stuiver et al., 1998). For the three marine sediment cores, Marine13 was used for calibration data and curve selection, and IntCal13 was used for the Tagus River Floodplain core (Reimer et al., 2013). All radiocarbon dates mentioned have age spans in the $2 \sigma$ range and are expressed as calibrated ages (cal BP; Table 2, Fig. S2).

\subsection{Bulk isotope data}

Prior to bulk carbon isotope analysis, sediment was decalcified using a $2 \mathrm{~N}$ HCL solution for approximately $18 \mathrm{~h}$. The sediment was rinsed three times using double-distilled water and then freeze-dried again. Total organic carbon (TOC) and $\delta^{13} \mathrm{C}_{\mathrm{TOC}}$ (Table 3) were measured in duplicate using a Flash 2000 series organic elemental analyzer (Thermo Scientific) 
Table 1. Stations, sediment core names, locations of sampling, and water depth for each sediment core used in this study.

\begin{tabular}{llccc}
\hline Station & Core name & Latitude $(\mathrm{N})$ & Longitude $(\mathrm{W})$ & Water depth $(\mathrm{m})$ \\
\hline 0501.029 & Tagus River Floodplain & $39^{\circ} 23^{\prime} 07.80^{\prime \prime}$ & $08^{\circ} 31^{\prime} 55.56^{\prime \prime}$ & 0 \\
64PE332-30-2 & Tagus Mudbelt & $38^{\circ} 39^{\prime} 02.20^{\prime \prime}$ & $09^{\circ} 28^{\prime} 07.68^{\prime \prime}$ & 82 \\
64PE332-44-2 & Lisbon Canyon Head & $38^{\circ} 30^{\prime} 20.19^{\prime \prime}$ & $09^{\circ} 15^{\prime} 04.87^{\prime \prime}$ & 259 \\
64PE269-39-4 & Lower Setúbal Canyon & $38^{\circ} 13^{\prime} 12.00^{\prime \prime}$ & $10^{\circ} 10^{\prime} 00.00^{\prime \prime}$ & 4217 \\
\hline
\end{tabular}

Table 2. Summary of the data used to determine an age depth model for the sediment samples in this study.

\begin{tabular}{|c|c|c|c|c|c|c|c|c|}
\hline Sediment core & Lab code & $\begin{array}{l}\text { Depth in } \\
\text { core }(\mathrm{cm})\end{array}$ & $\begin{array}{l}\text { Mean depth } \\
\text { in core }(\mathrm{cm})\end{array}$ & $\begin{array}{r}\text { Uncorrected } \\
\mathrm{AMS}^{14} \mathrm{C} \\
\text { ages }(\mathrm{yr} \mathrm{BP})\end{array}$ & $\begin{array}{r}\text { Analytical } \\
\text { error }( \pm 1 \sigma) \\
(\text { years })\end{array}$ & $\begin{array}{r}\text { Ages } \\
(\Delta \mathrm{R}=0 \mathrm{yr}) \\
( \pm 2 \sigma)(\text { cal yr BP })\end{array}$ & $\begin{array}{r}\text { Ages } \\
\text { (cal yr BP) }\end{array}$ & Analyzed material \\
\hline 0501.029* & & $0-2$ & 1 & & & & 0 & \\
\hline $0501.029^{*}$ & & $331-334$ & 332.5 & 1136 & 38 & $964-1150$ & 1057 & Roots of fraction $>125 \mu \mathrm{m}$ \\
\hline $0501.029^{*}$ & & $331-334$ & 332.5 & 1022 & 37 & $901-1001$ & 951 & Total organic fraction $>125 \mu \mathrm{m}$ \\
\hline $0501.029^{*}$ & & $604-607$ & 605.5 & 3089 & 38 & $3209-3383$ & 3296 & Terrestrial botanical macrofossils \\
\hline $0501.029^{*}$ & & $711-712$ & 711.5 & 4129 & 42 & $4530-4821$ & 4676 & Terrestrial botanical macrofossils \\
\hline $0501.029^{*}$ & & $1024-1029$ & 1026.5 & 5790 & 40 & $6485-6676$ & 6581 & Terrestrial botanical macrofossils \\
\hline 0501.029* & & $1046-1050$ & 1048 & 5900 & 45 & $6633-6805$ & 6719 & Terrestrial botanical macrofossils \\
\hline 64PE332-30-2 & & $0-2$ & 1 & & & & 0 & \\
\hline 64PE332-30-2 & BETA 348791 & $20-22$ & 21 & 500 & 30 & $40-236$ & 138 & Gastropod fragments \\
\hline 64PE332-30-2 & BETA 348792 & $428-430$ & 429 & 1730 & 30 & $1221-1349$ & 1285 & Ammonia beccarii (benthic forams) \\
\hline 64PE332-30-2 & BETA 348793 & $678-680$ & 679 & 2320 & 30 & $1848-2032$ & 1940 & Gastropod \\
\hline 64PE332-30-2 & BETA 317911 & $976-978$ & 977 & 5370 & 30 & $5643-5849$ & 5746 & Bivalve shell fragments \\
\hline 64PE332-44-2 & & $0-2$ & 1 & & & & 0 & \\
\hline 64PE332-44-2 & BETA 317906 & $521-523$ & 521.5 & 2330 & 30 & $1858-2044$ & 1951 & Mixed planktonic forams \\
\hline 64PE332-44-2 & BETA 317907 & $770-772$ & 771.5 & 5390 & 30 & $5664-5865$ & 5765 & Gastropod \\
\hline 64PE332-44-2 & BETA 317908 & $924.5-926.5$ & 925.5 & 8160 & 40 & $8515-8798$ & 8657 & Mixed planktonic forams \\
\hline 64PE269-39-4 & & 0 & 0 & & & & 0 & \\
\hline 64PE269-39-4 & BETA 330562 & 5 & 5 & 930 & 30 & $486-608$ & 547 & G. bulloides (planktonic forams) \\
\hline 64PE269-39-4 & BETA 330563 & 100 & 100 & 4980 & 50 & $5205-5466$ & 5336 & G. bulloides (planktonic forams) \\
\hline 64PE269-39-4 & BETA 330564 & 200 & 200 & 10190 & 40 & $11092-11271$ & 11182 & G. bulloides (planktonic forams) \\
\hline 64PE269-39-4 & BETA 348794 & 280 & 280 & 11540 & 40 & $12865-13150$ & 13008 & G. bulloides (planktonic forams) \\
\hline
\end{tabular}

* Data are from Vis et al. (2010)

equipped with a TCD detector. The $\delta^{13} \mathrm{C}_{\mathrm{TOC}}$ is expressed in relation to the Vienna Pee Dee Belemnite (VPDB) standard, and the isotope analysis precision was $0.1 \%$.

\subsection{Lipid extraction and GDGT analysis}

Between 1 and $3 \mathrm{~g}$ of freeze-dried sediment was extracted using Dionex ${ }^{\mathrm{TM}}$ accelerated solvent extraction (ASE) with dichloromethane (DCM) : methanol $(9: 1, v / v)$ as the solvent at a temperature of $100{ }^{\circ} \mathrm{C}$ and a pressure of $1500 \mathrm{psi}$ for $5 \mathrm{~min}$ with $60 \%$ flush and $60 \mathrm{~s}$ of purge. The extract was then collected and dried using a Caliper Turbovap ${ }^{\circledR}$ LV. Next, using DCM, the lipid extract was dried over a column of anhydrous $\mathrm{Na}_{2} \mathrm{SO}_{4}$ and then blown down under a gentle stream of $\mathrm{N}_{2}$. In order to quantify GDGTs, $1 \mu \mathrm{g}$ of an internal standard ( $\mathrm{C}_{46}$ GDGT; Huguet et al., 2006) was added to the total lipid extract before it was separated over a column of $\mathrm{Al}_{2} \mathrm{O}_{3}$ (activated for $2 \mathrm{~h}$ at $150^{\circ} \mathrm{C}$ ) into three fractions using hexane: DCM $(9: 1, v: v)$ for the apolar fraction, hexane $\operatorname{DCM}(1: 1, v: v)$ for the ketone fraction, and $\mathrm{DCM}: \mathrm{MeOH}(1: 1, v: v)$ for the polar fraction. The polar fraction, which contained the GDGTs, was dried under a $\mathrm{N}_{2}$ stream and then re-dissolved in hexane: isopropanol $(99: 1, v: v)$ at a concentration $10 \mathrm{mg} \mathrm{mL}^{-1}$. Finally, it was passed through a $0.45 \mu \mathrm{m}$ PTFE filter and analyzed with high-performance liquid chromatography-atmospheric pressure positive ion chemical ionization mass spectrometry (HPLC-APCI-MS) with a separation method that allows the separation of 5- and 6-methyl brGDGTs (Hopmans et al., 2015). For the study of Zell et al. (2014) the samples were split into two different fractions before the analysis: the intact polar lipid (IPL) fraction and core lipid (CL) fractions. For the purposes of this study the IPL and CL fractions of the river SPM were analyzed separately on the HPLC-APCI-MS for GDGTs (Hopmans et al., 2015) and then the amounts of GDGTs found in the CL and IPL fractions were combined. After analysis, some of the GDGT-based indices were recalculated for the entire sample set.

\subsection{Calculation of GDGT-based proxies}

The Roman numerals used to calculate the following GDGTbased proxies refer to the GDGTs indicated in Fig. S1. The GDGT indicated by IV is crenarchaeol, the isoprenoid 
Table 3. Concentrations of GDGTs and brGDGT-based indices for each sample set along the transect.

\begin{tabular}{|c|c|c|c|c|c|c|c|c|c|c|c|}
\hline \multirow[t]{2}{*}{ Sample name } & \multirow{2}{*}{$\begin{array}{l}\text { Age } \\
\text { (cal kyr BP) }\end{array}$} & \multirow{2}{*}{$\begin{array}{l}\text { TOC }^{*} \\
(\mathrm{wt} \%)\end{array}$} & \multirow{2}{*}{$\begin{array}{r}\delta^{13} \mathrm{C}_{\mathrm{TOC}}^{*} \\
(\% \circ \mathrm{VPDB})\end{array}$} & \multicolumn{2}{|c|}{ Concentration } & \multirow{2}{*}{$\begin{array}{r}\text { BIT } \\
\text { index }\end{array}$} & \multirow[t]{2}{*}{$\mathrm{MBT}_{5 \mathrm{me}}$} & \multirow[t]{2}{*}{ DC' } & \multirow[t]{2}{*}{ IR } & \multirow[t]{2}{*}{$\mathrm{IR}_{\mathrm{II}}$} & \multirow[t]{2}{*}{$\mathrm{IR}_{\mathrm{III}}$} \\
\hline & & & & $\left(\mu \mathrm{g} \mathrm{OC}^{-1}\right)$ & $\begin{array}{l}\text { Sum } \\
\text { brGDGTs } \\
-1)\end{array}$ & & & & & & \\
\hline \multicolumn{12}{|l|}{ Tagus soils } \\
\hline TRS-8b & $\mathrm{n} / \mathrm{a}$ & 3.0 & -27.8 & 2.2 & 19.2 & 0.88 & 0.51 & 0.13 & 0.39 & 0.36 & 0.43 \\
\hline TRS-7 & $\mathrm{n} / \mathrm{a}$ & 5.0 & -27.5 & 0.0 & 7.5 & 1.00 & 0.56 & 0.01 & 0.13 & 0.12 & 0.17 \\
\hline TRS-9 & $\mathrm{n} / \mathrm{a}$ & 0.5 & -27.2 & 0.1 & 7.8 & 0.99 & 0.68 & 0.01 & 0.14 & 0.14 & 0.15 \\
\hline TRS-3 & $\mathrm{n} / \mathrm{a}$ & 0.7 & -29.0 & 0.6 & 4.1 & 0.87 & 0.40 & 0.05 & 0.41 & 0.43 & 0.39 \\
\hline TRS-4 & $\mathrm{n} / \mathrm{a}$ & 0.7 & -28.7 & 1.2 & 5.3 & 0.81 & 0.42 & 0.04 & 0.39 & 0.41 & 0.30 \\
\hline TRS-5 & $\mathrm{n} / \mathrm{a}$ & 2.2 & -28.4 & 2.4 & 15.3 & 0.85 & 0.29 & 0.10 & 0.37 & 0.37 & 0.35 \\
\hline TRS-10 & $\mathrm{n} / \mathrm{a}$ & 1.5 & -28.5 & 1.4 & 1.6 & 0.49 & 0.60 & 0.19 & 0.84 & 0.88 & 0.83 \\
\hline TRS-12 & $\mathrm{n} / \mathrm{a}$ & 0.2 & -25.1 & 4.0 & 2.4 & 0.34 & 0.62 & 0.22 & 0.87 & 0.87 & 0.91 \\
\hline TRS-14b & $\mathrm{n} / \mathrm{a}$ & 0.8 & -25.3 & 1.7 & 2.1 & 0.48 & 0.57 & 0.32 & 0.85 & 0.89 & 0.86 \\
\hline TRS-13 & $\mathrm{n} / \mathrm{a}$ & 6.9 & -27.0 & 0.9 & 1.2 & 0.54 & 0.33 & 0.13 & 0.74 & 0.80 & 0.71 \\
\hline TRS-15 & $\mathrm{n} / \mathrm{a}$ & 0.9 & -25.7 & 1.9 & 2.0 & 0.48 & 0.46 & 0.21 & 0.86 & 0.90 & 0.86 \\
\hline TRS-16 & $\mathrm{n} / \mathrm{a}$ & 0.1 & -24.8 & 1.6 & 3.0 & 0.62 & 0.56 & 0.19 & 0.92 & 0.93 & 0.93 \\
\hline TRS-20 & $\mathrm{n} / \mathrm{a}$ & 0.1 & -26.1 & 0.6 & 3.8 & 0.84 & 0.52 & 0.38 & 0.83 & 0.87 & 0.83 \\
\hline TRS-19 & $\mathrm{n} / \mathrm{a}$ & 0.1 & -25.7 & 0.7 & 19.7 & 0.95 & 0.56 & 0.44 & 0.84 & 0.85 & 0.89 \\
\hline \multicolumn{12}{|c|}{ Tagus riverbank sediments } \\
\hline TRS-6 & $\mathrm{n} / \mathrm{a}$ & 1.7 & -26.3 & 16.7 & 76.0 & 0.77 & 0.53 & 0.30 & 0.54 & 0.46 & 0.59 \\
\hline TRS-8a & $\mathrm{n} / \mathrm{a}$ & 0.2 & -26.5 & 10.8 & 68.2 & 0.83 & 0.55 & 0.26 & 0.77 & 0.71 & 0.83 \\
\hline TRS 2a & $\mathrm{n} / \mathrm{a}$ & 3.3 & -23.6 & 3.4 & 12.8 & 0.70 & 0.63 & 0.42 & 0.79 & 0.81 & 0.77 \\
\hline TRS $2 b$ & $\mathrm{n} / \mathrm{a}$ & 0.5 & -25.1 & 8.1 & 55.1 & 0.80 & 0.57 & 0.45 & 0.67 & 0.72 & 0.72 \\
\hline TRS 1a & $\mathrm{n} / \mathrm{a}$ & 0.9 & -26.9 & 9.7 & 18.8 & 0.62 & 0.54 & 0.21 & 0.68 & 0.68 & 0.66 \\
\hline TRS1b & $\mathrm{n} / \mathrm{a}$ & 1.5 & -27.6 & 26.1 & 19.7 & 0.28 & 0.64 & 0.53 & 0.72 & 0.82 & 0.80 \\
\hline TRS-11 & $\mathrm{n} / \mathrm{a}$ & 1.3 & -27.0 & 6.3 & 3.9 & 0.33 & 0.48 & 0.24 & 0.75 & 0.79 & 0.75 \\
\hline TRS-14a & $\mathrm{n} / \mathrm{a}$ & 3.0 & -27.1 & 1.2 & 21.7 & 0.92 & 0.38 & 0.44 & 0.55 & 0.56 & 0.68 \\
\hline TRS-17 & $\mathrm{n} / \mathrm{a}$ & 3.7 & -29.6 & 1.6 & 37.8 & 0.93 & 0.41 & 0.51 & 0.70 & 0.65 & 0.66 \\
\hline TRS-22 & $\mathrm{n} / \mathrm{a}$ & 0.6 & -29.8 & 3.1 & 24.8 & 0.86 & 0.41 & 0.40 & 0.80 & 0.75 & 0.82 \\
\hline \multicolumn{12}{|l|}{ Tagus River SPM } \\
\hline TR 2 Sup July & $\mathrm{n} / \mathrm{a}$ & 2.6 & -29.2 & 6.2 & 33.1 & 0.80 & 0.51 & 0.28 & 0.60 & 0.53 & 0.64 \\
\hline TR 3\#1 Sup Sept. & $\mathrm{n} / \mathrm{a}$ & 1.8 & -28.4 & 5.6 & 20.7 & 0.73 & 0.52 & 0.30 & 0.59 & 0.51 & 0.61 \\
\hline TR4 \#1 Oct. & $\mathrm{n} / \mathrm{a}$ & 2.5 & -30.9 & 8.6 & 38.1 & 0.77 & 0.52 & 0.30 & 0.61 & 0.54 & 0.62 \\
\hline TR5 \#1 Sup Nov. & $\mathrm{n} / \mathrm{a}$ & 1.3 & -28.9 & 5.8 & 54.8 & 0.85 & 0.49 & 0.25 & 0.62 & 0.55 & 0.68 \\
\hline TR 6 \#1 Sup Dec. & $\mathrm{n} / \mathrm{a}$ & 2.4 & -29.4 & 11.5 & 86.8 & 0.85 & 0.47 & 0.26 & 0.63 & 0.56 & 0.67 \\
\hline TR7 \#1 Sup Jan. & $\mathrm{n} / \mathrm{a}$ & 2.4 & -29.8 & 9.8 & 53.6 & 0.77 & 0.48 & 0.27 & 0.63 & 0.56 & 0.67 \\
\hline TR8 \#1 Sup Feb. & $\mathrm{n} / \mathrm{a}$ & 1.0 & -29.4 & 16.8 & 46.9 & 0.69 & 0.50 & 0.27 & 0.58 & 0.51 & 0.62 \\
\hline TR9 \#1 Sup Mar. & $\mathrm{n} / \mathrm{a}$ & 2.2 & -29.0 & 6.3 & 21.9 & 0.72 & 0.50 & 0.29 & 0.58 & 0.51 & 0.61 \\
\hline TR10 \#1 Sup Apr. & $\mathrm{n} / \mathrm{a}$ & 1.9 & -28.5 & 0.8 & 36.5 & 0.96 & 0.52 & 0.29 & 0.58 & 0.50 & 0.61 \\
\hline TR11 \#1 Sup May & $\mathrm{n} / \mathrm{a}$ & 1.7 & -28.5 & 26.3 & 80.8 & 0.69 & 0.52 & 0.29 & 0.56 & 0.48 & 0.59 \\
\hline TR12 \#1 Sup June & $\mathrm{n} / \mathrm{a}$ & 1.3 & -27.8 & 9.5 & 22.8 & 0.65 & 0.55 & 0.23 & 0.51 & 0.48 & 0.58 \\
\hline $\begin{array}{l}\text { Tagus River Floodp } \\
\text { sediments }(0501.02\end{array}$ & & & & & & & & & & & \\
\hline 10.0 & 0.0 & 1.7 & -26.3 & 2.1 & 29.7 & 0.92 & 0.34 & 0.31 & 0.39 & 0.39 & 0.44 \\
\hline 95.0 & 0.3 & 2.6 & -26.5 & 6.4 & 66.8 & 0.89 & 0.36 & 0.24 & 0.43 & 0.43 & 0.45 \\
\hline 195.0 & 0.6 & 1.5 & -27.4 & 2.5 & 111.8 & 0.97 & 0.34 & 0.37 & 0.38 & 0.40 & 0.43 \\
\hline 241.0 & 0.8 & 1.5 & -26.0 & 5.2 & 62.0 & 0.90 & 0.40 & 0.24 & 0.35 & 0.36 & 0.38 \\
\hline 341.5 & 1.0 & 11.2 & -27.5 & 0.9 & 47.6 & 0.97 & 0.45 & 0.39 & 0.36 & 0.34 & 0.37 \\
\hline 401.0 & 1.5 & 12.1 & -27.7 & 1.2 & 90.6 & 0.98 & 0.38 & 0.38 & 0.40 & 0.36 & 0.46 \\
\hline 453.0 & 2.0 & 8.0 & -26.7 & 3.0 & 120.9 & 0.97 & 0.34 & 0.35 & 0.43 & 0.43 & 0.50 \\
\hline 542.0 & 2.8 & 7.7 & -27.3 & 3.2 & 91.9 & 0.95 & 0.37 & 0.39 & 0.40 & 0.41 & 0.42 \\
\hline 577.0 & 3.1 & 4.6 & -27.2 & 3.3 & 84.2 & 0.95 & 0.43 & 0.35 & 0.38 & 0.37 & 0.41 \\
\hline 641.0 & 3.8 & 5.3 & -28.1 & 1.0 & 59.6 & 0.97 & 0.52 & 0.39 & 0.34 & 0.35 & 0.39 \\
\hline 681.0 & 4.3 & 6.6 & -28.6 & 1.0 & 52.3 & 0.97 & 0.46 & 0.45 & 0.37 & 0.37 & 0.42 \\
\hline 741.0 & 4.9 & 4.2 & -24.4 & 4.2 & 74.3 & 0.92 & 0.43 & 0.45 & 0.38 & 0.38 & 0.36 \\
\hline 862.0 & 5.6 & 16.2 & -26.8 & 2.2 & 58.4 & 0.94 & 0.45 & 0.45 & 0.46 & 0.43 & 0.52 \\
\hline 982.0 & 6.3 & 8.7 & -27.7 & 1.5 & 46.5 & 0.95 & 0.46 & 0.49 & 0.37 & 0.36 & 0.37 \\
\hline 1041.0 & 6.7 & 5.2 & -27.2 & 4.4 & 48.2 & 0.87 & 0.48 & 0.45 & 0.40 & 0.39 & 0.41 \\
\hline
\end{tabular}


Table 3. Continued.

\begin{tabular}{|c|c|c|c|c|c|c|c|c|c|c|c|}
\hline \multirow[t]{2}{*}{ Sample name } & \multirow[t]{2}{*}{$\begin{array}{l}\text { Age } \\
\text { (cal kyr BP) }\end{array}$} & \multirow[t]{2}{*}{$\begin{array}{l}\text { TOC }^{*} \\
(\mathrm{wt} \%)\end{array}$} & \multirow[t]{2}{*}{$\begin{array}{c}\delta^{13} \mathrm{C}_{\mathrm{TOC}}^{*} \\
(\% \circ \mathrm{VPDB})\end{array}$} & \multicolumn{2}{|c|}{ Concentration } & \multirow[t]{2}{*}{$\begin{array}{r}\text { BIT } \\
\text { index }\end{array}$} & \multirow[t]{2}{*}{$\mathrm{MBT}^{\prime}{ }_{5 \mathrm{me}}$} & \multirow[t]{2}{*}{ DC' } & \multirow[t]{2}{*}{ IR } & \multirow[t]{2}{*}{$\mathrm{IR}_{\mathrm{II}}$} & \multirow[t]{2}{*}{$\mathrm{IR}_{\mathrm{III}}$} \\
\hline & & & & $\begin{array}{l}\text { Crenarchaeol } \\
\qquad(\mu \mathrm{gg} \mathrm{O}\end{array}$ & $\begin{array}{l}\text { Sum } \\
\text { rGDGT } \\
\text { l) }\end{array}$ & & & & & & \\
\hline $\begin{array}{l}\text { Mudbelt sedim } \\
\text { (64PE332-30-2 }\end{array}$ & & & & & & & & & & & \\
\hline 1.0 & 0.0 & 1.2 & -23.7 & 200.9 & 28.5 & 0.09 & 0.58 & 0.35 & 0.51 & 0.45 & 0.54 \\
\hline 25.0 & 0.2 & 1.0 & -24.2 & 194.7 & 40.7 & 0.13 & 0.54 & 0.28 & 0.49 & 0.43 & 0.52 \\
\hline 53.0 & 0.2 & 0.9 & -24.5 & 189.3 & 36.4 & 0.13 & 0.52 & 0.26 & 0.47 & 0.44 & 0.50 \\
\hline 75.0 & 0.3 & 1.0 & -24.4 & 206.5 & 43.8 & 0.14 & 0.52 & 0.26 & 0.48 & 0.43 & 0.51 \\
\hline 101.0 & 0.4 & 0.9 & -24.3 & 228.9 & 44.4 & 0.13 & 0.53 & 0.27 & 0.48 & 0.43 & 0.51 \\
\hline 151.0 & 0.5 & 0.9 & -24.3 & 194.8 & 40.2 & 0.13 & 0.53 & 0.27 & 0.49 & 0.44 & 0.53 \\
\hline 201.0 & 0.6 & 1.0 & -24.5 & 187.9 & 31.9 & 0.11 & 0.51 & 0.27 & 0.47 & 0.43 & 0.50 \\
\hline 248.0 & 0.8 & 0.6 & -24.5 & 224.0 & 58.0 & 0.14 & 0.53 & 0.26 & 0.46 & 0.42 & 0.49 \\
\hline 297.0 & 0.9 & 1.1 & -24.5 & 168.3 & 30.6 & 0.12 & 0.50 & 0.26 & 0.47 & 0.42 & 0.51 \\
\hline 347.0 & 1.1 & 1.0 & -24.1 & 202.5 & 24.3 & 0.08 & 0.52 & 0.30 & 0.50 & 0.45 & 0.53 \\
\hline 397.0 & 1.2 & 1.1 & -24.1 & 180.4 & 27.3 & 0.10 & 0.49 & 0.30 & 0.48 & 0.43 & 0.52 \\
\hline 429.0 & 1.3 & 1.2 & -24.0 & 77.3 & 8.7 & 0.08 & 0.50 & 0.34 & 0.53 & 0.45 & 0.57 \\
\hline 496.0 & 1.5 & 1.0 & -24.3 & 143.8 & 15.1 & 0.07 & 0.52 & 0.32 & 0.53 & 0.46 & 0.57 \\
\hline 546.0 & 1.6 & 0.9 & -24.1 & 11.2 & 1.4 & 0.08 & 0.52 & 0.33 & 0.51 & 0.45 & 0.55 \\
\hline 596.0 & 1.7 & 1.1 & -27.3 & 141.3 & 14.8 & 0.07 & 0.51 & 0.34 & 0.52 & 0.45 & 0.56 \\
\hline 645.0 & 1.9 & 1.1 & -29.9 & 108.7 & 12.8 & 0.08 & 0.52 & 0.34 & 0.52 & 0.45 & 0.55 \\
\hline 680.0 & 2.0 & 1.1 & -27.4 & 142.1 & 17.4 & 0.08 & 0.54 & 0.31 & 0.50 & 0.44 & 0.54 \\
\hline 741.0 & 2.7 & 0.8 & -27.3 & 176.1 & 16.0 & 0.06 & 0.52 & 0.35 & 0.53 & 0.46 & 0.55 \\
\hline 791.0 & 3.4 & 0.7 & -24.5 & 240.8 & 19.8 & 0.05 & 0.50 & 0.37 & 0.55 & 0.48 & 0.58 \\
\hline 840.0 & 4.0 & 0.9 & -24.4 & 139.3 & 11.0 & 0.05 & 0.48 & 0.37 & 0.54 & 0.48 & 0.55 \\
\hline 890.0 & 4.6 & 0.6 & -24.6 & 180.2 & 14.2 & 0.05 & 0.48 & 0.39 & 0.55 & 0.47 & 0.57 \\
\hline 977.0 & 5.7 & 0.7 & -27.5 & 125.8 & 11.8 & 0.06 & 0.46 & 0.40 & 0.53 & 0.46 & 0.52 \\
\hline $\begin{array}{l}\text { Lisbon Canyon } \\
\text { (64PE332-44-2 }\end{array}$ & & & & & & & & & & & \\
\hline 1.0 & 0.0 & 1.5 & -22.9 & 420.5 & 27.1 & 0.04 & 0.60 & 0.41 & 0.55 & 0.48 & 0.56 \\
\hline 45.0 & 0.2 & 1.2 & -22.8 & 409.9 & 47.3 & 0.07 & 0.58 & 0.40 & 0.55 & 0.48 & 0.57 \\
\hline 85.0 & 0.3 & 1.1 & -23.4 & 440.4 & 48.1 & 0.07 & 0.54 & 0.36 & 0.53 & 0.46 & 0.55 \\
\hline 130.5 & 0.5 & 1.2 & -23.4 & 429.6 & 41.2 & 0.06 & 0.55 & 0.36 & 0.53 & 0.46 & 0.57 \\
\hline 187.5 & 0.7 & 1.2 & -23.7 & 308.4 & 27.6 & 0.06 & 0.56 & 0.36 & 0.53 & 0.45 & 0.56 \\
\hline 221.5 & 0.8 & 1.1 & -24.4 & 157.9 & 35.1 & 0.12 & 0.54 & 0.28 & 0.47 & 0.42 & 0.51 \\
\hline 278.5 & 1.0 & 1.2 & -23.2 & 283.6 & 22.7 & 0.05 & 0.54 & 0.36 & 0.51 & 0.45 & 0.54 \\
\hline 326.5 & 1.2 & 1.0 & -22.6 & 361.7 & 31.1 & 0.05 & 0.53 & 0.42 & 0.56 & 0.47 & 0.60 \\
\hline 371.5 & 1.4 & 1.1 & -22.6 & 374.1 & 26.5 & 0.05 & 0.53 & 0.41 & 0.55 & 0.47 & 0.58 \\
\hline 429.0 & 1.6 & 1.0 & -22.6 & 399.6 & 29.2 & 0.05 & 0.55 & 0.42 & 0.56 & 0.49 & 0.59 \\
\hline 480.0 & 1.8 & 1.1 & -23.4 & 242.3 & 18.0 & 0.05 & 0.54 & 0.41 & 0.55 & 0.48 & 0.57 \\
\hline 502.0 & 1.9 & 1.1 & -23.9 & 365.1 & 25.4 & 0.04 & 0.52 & 0.42 & 0.56 & 0.49 & 0.58 \\
\hline 522.0 & 2.0 & 1.0 & -23.4 & 376.8 & 30.9 & 0.05 & 0.55 & 0.39 & 0.55 & 0.48 & 0.57 \\
\hline 550.0 & 2.4 & 1.0 & -23.0 & 363.1 & 26.7 & 0.05 & 0.54 & 0.43 & 0.57 & 0.50 & 0.59 \\
\hline 570.0 & 2.7 & 0.7 & -22.6 & 493.6 & 34.3 & 0.04 & 0.53 & 0.43 & 0.57 & 0.50 & 0.59 \\
\hline 630.0 & 3.6 & 0.7 & -22.4 & 389.6 & 25.7 & 0.04 & 0.53 & 0.44 & 0.58 & 0.51 & 0.60 \\
\hline 686.0 & 4.5 & 0.5 & -22.4 & 640.2 & 40.7 & 0.04 & 0.54 & 0.45 & 0.59 & 0.51 & 0.60 \\
\hline 728.0 & 5.1 & 0.5 & -22.1 & 100.1 & 6.7 & 0.04 & 0.50 & 0.45 & 0.57 & 0.51 & 0.58 \\
\hline 771.0 & 5.8 & 0.7 & -23.3 & 620.7 & 37.3 & 0.04 & 0.53 & 0.44 & 0.58 & 0.50 & 0.59 \\
\hline 805.0 & 6.4 & 0.3 & -22.1 & 414.0 & 25.9 & 0.04 & 0.52 & 0.45 & 0.58 & 0.51 & 0.58 \\
\hline 869.5 & 7.6 & 0.3 & -22.3 & 538.9 & 35.7 & 0.04 & 0.54 & 0.41 & 0.57 & 0.50 & 0.56 \\
\hline 925.5 & 8.7 & 0.3 & -23.1 & 409.4 & 30.1 & 0.04 & 0.52 & 0.46 & 0.59 & 0.51 & 0.58 \\
\hline $\begin{array}{l}\text { Lower Setúbal } \\
\text { sediments }(64 \mathrm{P}\end{array}$ & & & & & & & & & & & \\
\hline 1.0 & 0.0 & 0.8 & -23.5 & 265.3 & 20.3 & 0.05 & 0.68 & 0.38 & 0.73 & 0.60 & 0.77 \\
\hline 20.0 & 1.3 & 0.7 & -22.3 & 506.5 & 15.6 & 0.02 & 0.60 & 0.39 & 0.77 & 0.60 & 0.80 \\
\hline 40.0 & 2.3 & 0.6 & -22.0 & 751.5 & 18.1 & 0.02 & 0.58 & 0.39 & 0.73 & 0.61 & 0.75 \\
\hline 60.0 & 3.3 & 0.8 & -22.2 & 540.7 & 16.2 & 0.02 & 0.56 & 0.46 & 0.75 & 0.61 & 0.74 \\
\hline 80.0 & 4.3 & 0.8 & -22.3 & 460.5 & 13.5 & 0.02 & 0.56 & 0.41 & 0.75 & 0.63 & 0.77 \\
\hline 100.0 & 5.3 & 0.8 & -22.8 & 204.7 & 7.3 & 0.03 & 0.57 & 0.37 & 0.74 & 0.66 & 0.75 \\
\hline 120.0 & 6.5 & 0.7 & -22.8 & 199.2 & 8.0 & 0.03 & 0.57 & 0.38 & 0.77 & 0.66 & 0.78 \\
\hline 140.0 & 7.7 & 0.5 & -26.1 & 433.3 & 13.6 & 0.02 & 0.51 & 0.41 & 0.73 & 0.67 & 0.74 \\
\hline 160.0 & 8.8 & 0.5 & -22.8 & 395.6 & 16.9 & 0.03 & 0.45 & 0.43 & 0.69 & 0.64 & 0.64 \\
\hline 180.0 & 10.0 & 0.4 & -24.9 & 709.2 & 25.6 & 0.02 & 0.48 & 0.43 & 0.69 & 0.58 & 0.65 \\
\hline 200.0 & 11.2 & 0.5 & -25.7 & 703.9 & 22.1 & 0.02 & 0.54 & 0.41 & 0.66 & 0.52 & 0.64 \\
\hline 220.0 & 11.6 & $\mathrm{n} / \mathrm{a}$ & $\mathrm{n} / \mathrm{a}$ & $\mathrm{n} / \mathrm{a}$ & $\mathrm{n} / \mathrm{a}$ & 0.02 & 0.48 & 0.47 & 0.66 & 0.52 & 0.59 \\
\hline 240.0 & 12.1 & $\mathrm{n} / \mathrm{a}$ & $\mathrm{n} / \mathrm{a}$ & $\mathrm{n} / \mathrm{a}$ & $\mathrm{n} / \mathrm{a}$ & 0.01 & 0.56 & 0.57 & 0.70 & 0.56 & 0.63 \\
\hline 260.0 & 12.6 & $\mathrm{n} / \mathrm{a}$ & $\mathrm{n} / \mathrm{a}$ & $\mathrm{n} / \mathrm{a}$ & $\mathrm{n} / \mathrm{a}$ & 0.03 & 0.58 & 0.49 & 0.71 & 0.58 & 0.64 \\
\hline 280.0 & 13.0 & $\mathrm{n} / \mathrm{a}$ & $\mathrm{n} / \mathrm{a}$ & $\mathrm{n} / \mathrm{a}$ & $\mathrm{n} / \mathrm{a}$ & 0.01 & 0.52 & 0.48 & 0.69 & 0.57 & 0.65 \\
\hline
\end{tabular}

* Data for Tagus soils, riverbank sediments, and SPM have previously been published by Zell et al. (2014). 
GDGT specific to Thaumarchaeota (Sinninghe Damsté et al., 2002).

The BIT index (Hopmans et al., 2003), which results in a value between 0 and 1 , with those values closer to 0 designating a more marine signal and a value close to 1 indicating a more terrestrial signal, was calculated using the following formulae that specifically include the novel 6-methyl brGDGTs according to De Jonge et al. (2015):

$$
\begin{array}{r}
\text { BIT index }=\left(\mathrm{Ia}+\mathrm{IIa}+\mathrm{IIIa}+\mathrm{IIa}^{\prime}+\mathrm{IIIa}^{\prime}\right) / \\
\left(\mathrm{Ia}+\mathrm{IIa}+\mathrm{IIIa}+\mathrm{IIa}^{\prime}+\mathrm{IIIa}^{\prime}+\mathrm{IV}\right)
\end{array}
$$

The isomer ratio (IR) signifies the quantity of the pentaand hexamethylated 6-methyl brGDGTs compared to the total brGDGTs and was calculated according to De Jonge et al. (2015):

$$
\begin{aligned}
& \mathrm{IR}=\left(\mathrm{IIa}^{\prime}+\mathrm{IIb}^{\prime}+\mathrm{IIc}^{\prime}+\mathrm{IIIa}^{\prime}+\mathrm{IIIb}^{\prime}+\mathrm{IIIc}^{\prime}\right) / \\
& \quad\left(\mathrm{IIa}+\mathrm{IIb}+\mathrm{IIc}+\mathrm{III}+\mathrm{III} \mathrm{II}+\mathrm{IIIc}+\mathrm{IIa}^{\prime}+\mathrm{IIb}^{\prime}+\mathrm{IIc}^{\prime}\right. \\
& \left.\quad+\mathrm{IIIa}^{\prime}+\mathrm{IIIb}^{\prime}+\mathrm{IIIc}^{\prime}\right) .
\end{aligned}
$$

The relative abundances of the penta- and hexamethylated 6methyl brGDGTs are calculated according to (De Jonge et al., 2014b):

$$
\begin{aligned}
\mathrm{IR}_{\mathrm{II}} & =\mathrm{IIa}^{\prime} /\left(\mathrm{II} \mathrm{a}+\mathrm{IIa}^{\prime}\right), \\
\mathrm{IR}_{\mathrm{III}} & =\mathrm{IIIa}^{\prime} /\left(\mathrm{III} \mathrm{I}+\mathrm{IIIa}^{\prime}\right) .
\end{aligned}
$$

The MBT' ${ }_{5 \mathrm{Me}}$ (which excludes the 6-methyl brGDGTs) was used to calculate MAT according to De Jonge et al. (2014a):

$$
\begin{gathered}
\mathrm{MBT}^{\prime}{ }_{5 \mathrm{Me}}=(\mathrm{Ia}+\mathrm{Ib}+\mathrm{Ic}) / \\
(\mathrm{Ia}+\mathrm{Ib}+\mathrm{Ic}+\mathrm{IIa}+\mathrm{IIb}+\mathrm{IIc}+\mathrm{IIIa}), \\
\mathrm{MAT}=-8.57+31.45 \times \mathrm{MBT}_{5 \mathrm{Me}}^{\prime} .
\end{gathered}
$$

The equation to determine DC (Sinninghe Damsté et al., 2009) was reformulated to specifically include the pentamethylated 6-methyl brGDGTs:

$$
\begin{aligned}
\mathrm{DC}^{\prime} & =\left(\mathrm{Ib}+\mathrm{IIb}+\mathrm{IIb}^{\prime}\right) / \\
& \left(\mathrm{Ia}+\mathrm{Ib}+\mathrm{IIa}+\mathrm{IIb}+\mathrm{IIa}^{\prime}+\mathrm{IIb}^{\prime}\right) .
\end{aligned}
$$

To calculate $\mathrm{pH}$ and MAT the novel $\mathrm{MAT}_{\mathrm{mr}} / \mathrm{CBT}$ ' calibration was used (De Jonge et al., 2014a):

$$
\begin{aligned}
\mathrm{CBT}^{\prime} & ={ }^{10} \log \left(\mathrm{Ic}+\mathrm{IIa}^{\prime}+\mathrm{IIb}^{\prime}+\mathrm{IIc}^{\prime}+\mathrm{IIIa}^{\prime}\right. \\
& \left.+\mathrm{IIIb}^{\prime}+\mathrm{Ic}^{\prime}\right) /(\mathrm{Ia}+\mathrm{IIa}+\mathrm{IIIa}), \\
\mathrm{pH} & =7.15+1.59 \times \mathrm{CBT}^{\prime}, \\
\mathrm{MAT}_{\mathrm{mr}} & =7.17+17.1 \times[\mathrm{Ia}] \\
& +25.9 \times[\mathrm{Ib}]+34.4 \times[\mathrm{Ic}]-28.6 \times[\mathrm{IIa}], \\
\mathrm{MAT}_{\mathrm{mrs}} & =5.58+17.91 \times[\mathrm{Ia}]-18.77 \times[\mathrm{II}] .
\end{aligned}
$$

\subsection{Statistical analysis}

Using the R software package for statistical analysis, we employed principal component analysis (PCA) based on the correlation matrix. The PCA was performed on the fractional abundances of all 15 of the 5- and 6-methyl brGDGTs for the entire sample set along the transect from the land to the ocean.

\section{Results}

We report bulk and brGDGT data for four cores covering Holocene sedimentation in the Tagus River basin and its outflow into the Atlantic. We compare these data with new results acquired through an improved LC method able to distinguish between the 5- and 6-methyl brGDGTs (De Jonge et al., 2013) on the soils, riverbank sediments, and SPM samples previously obtained by Zell et al. (2014).

\subsection{Bulk parameters of the sediments}

The age-depth models for the marine sediment cores (Fig. S2, Table 1) are based on radiocarbon dating of selected foraminifera, gastropods, and shell fragments. The data show that of the four sediment cores from the transect, the Tagus River Floodplain sediments date to $6.7 \mathrm{cal} \mathrm{kyr} \mathrm{BP}$, the Tagus Mudbelt sediments date to 5.8 cal kyr BP, the Lisbon Canyon Head sediments date to $8.7 \mathrm{cal} \mathrm{kyr} \mathrm{BP}$, and the Lower Setúbal Canyon penetrated the oldest strata $(13.0 \mathrm{cal}$ kyr BP). Reported values for sediments from each location were averaged over the interval 0-6.0 cal kyr BP so as to avoid a bias in the data since not all of the sediment cores covered more than $6.0 \mathrm{kyr}$.

The bulk carbon isotope data for the Tagus River SPM, riverbank sediments, and soils have been previously discussed in Zell et al. (2014). The TOC values for the Tagus River Floodplain sediments are relatively high and also highly variable, with a range of $1.5-16 \mathrm{wt} \%$ and a mean of $6.5 \pm 4.3 \mathrm{wt} \%$ (average \pm standard deviation), and the mean $\delta^{13} \mathrm{C}_{\mathrm{TOC}}$ was $-27.0 \pm 1.0 \%$ (Fig. 2; Table 3 ). In the Tagus Mudbelt sediments the TOC is less variable than in the Tagus River Floodplain sediments, ranging from 0.6 to $1.2 \mathrm{wt} \%$ and with an average of $0.9 \pm 0.2 \mathrm{wt} \%$ (Fig. 2; Table 3). The average $\delta^{13} \mathrm{C}_{\mathrm{TOC}}$ in the Tagus Mudbelt sediments, $-24.3 \pm 0.2 \%$, is higher than in the Tagus River Floodplain sediments. The average $\delta^{13} \mathrm{C}_{\text {TOC }}$ of the Lisbon Canyon Head sediments, $-23.0 \pm 0.6 \%$, is higher than the Tagus Mudbelt sediments and the TOC content is similar to that of the Tagus Mudbelt sediments, ranging from 0.25 to $1.5 \mathrm{wt} \%$ with the mean of $0.9 \pm 0.3 \mathrm{wt} \%$ (Fig. 2; Table 3). The average $\delta^{13} \mathrm{C}_{\mathrm{TOC}}$ values in the Lower Setúbal Canyon sediments $(-23.4 \pm 1.5 \%)$ are similar to those of the Lisbon Canyon Head sediments, with a TOC content ranging from 0.51 to $0.85 \mathrm{wt} \%$ with a mean value of $0.65 \pm 0.14 \mathrm{wt} \%$ (Fig. 2; Table 3). 
(a)

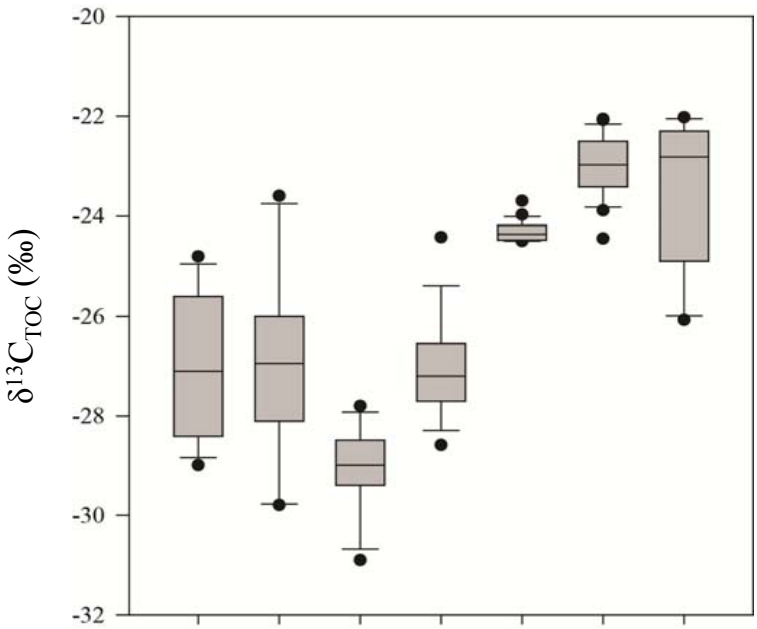

(b)

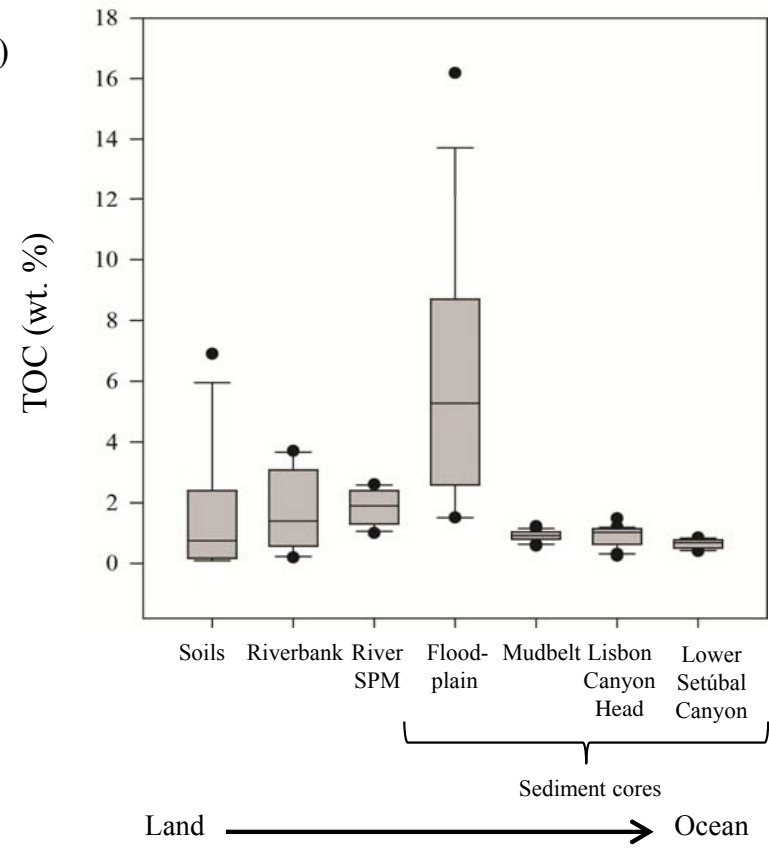

Figure 2. Box plots of (a) $\delta^{13} \mathrm{C}_{\mathrm{TOC}}(\%$ ) of the organic carbon and (b) TOC (wt \%) for each sample set along the Tagus River source to sink transect. The increasing $\delta^{13} \mathrm{C}_{\mathrm{TOC}}$ values in the sediment core locations with increasing distance from the coast indicate that more of the organic carbon in these sediments is marine-derived.

\subsection{Concentrations and distributions of GDGTs}

\subsubsection{Tagus soils and riverbank sediments}

The average concentration of crenarchaeol is higher in the riverbank sediments $\left(\sim 8.7 \pm 7.8 \mu \mathrm{g} \mathrm{g} \mathrm{OC}^{-1}\right)$ than in the soils $\left(\sim 1.4 \pm 1.1 \mu \mathrm{g} \mathrm{g} \mathrm{OC}^{-1}\right.$; Fig. 3a-b; Table 3$)$. The same trend is true for the brGDGTs, with the average concentration being higher in the riverbank sediments $\left(\sim 33.9 \pm 24.5 \mu \mathrm{g} \mathrm{gC}^{-1}\right)$ than the soils $\left(\sim 6.8 \pm 6.5 \mu \mathrm{g} \mathrm{g} \mathrm{OC}^{-1}\right.$; Fig. $3 \mathrm{a}-\mathrm{b}$; Table 3$)$. The values of the BIT index were similar to those previously reported (Zell et al., 2014) for both the soils and riverbank sediments and ranged from 0.3 to 1.0 with an average of $0.7 \pm 0.2$ (Fig. 3c; Table 3). The reanalysis of the brGDGTs in the soils reveals that the relative abundance of the novel 6-methyl brGDGTs is highly variable (ranging from 0.13 to 0.92 ) and can be quite high; the average values for the IR are $0.6 \pm 0.3$ (Fig. 3e; Table 3). IR is even higher but less variable for the riverbank sediments, with an average of $0.7 \pm 0.1$ (Fig. 3e; Table 3). In general the penta- and hexamethylated brGDGTs show the same ratio of 5- and 6-methyl isomers (Fig. 4); however, in soils from an altitude of $>350 \mathrm{~m}$ the 6-methyl brGDGTs are especially dominant (Fig. S3). Values for the new MBT'5me index, which excludes the 6-methyl brGDGTs (cf. De Jonge et al., 2014a), of the soils and riverbank sediments are quite similar, with an average of $0.5 \pm 0.1$ in both cases (Fig. 3f; Table 3). The DC' ratio deviates between the soils and the riverbank sediments (Fig. 3d; Table 3). The DC' for the soils is highly variable but on average low $(0.2 \pm 0.1)$; for the riverbank sediments it is higher with an average of $0.4 \pm 0.1$ (Fig. 3d; Table 3).

\subsubsection{Tagus River SPM}

The SPM was obtained from the Tagus Estuary near the mouth of the Tagus River once a month over the course of a year (excluding the month of August). Data from the Tagus River SPM showed that the summed brGDGT and crenarchaeol concentrations in the river SPM varied throughout the year and were on average $45 \pm 23$ and $9.8 \pm 6.8 \mu \mathrm{g} \mathrm{gCC}^{-1}$ (Fig. 3a-b; Table 3), respectively, resulting in only small variations in the BIT index (i.e., $0.8 \pm 0.1$; Fig. 3c; Table 3). The distribution of brGDGTs (Fig. 5c) was relatively constant throughout the year, as is evident from the values for

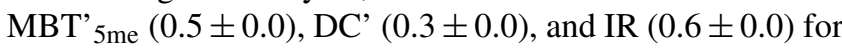
the river SPM (Fig. 3d-f; Table 3).

\subsubsection{Tagus River Floodplain sediments}

The average crenarchaeol concentration is fairly low in the Tagus River Floodplain sediments, $2.8 \pm 1.7 \mu \mathrm{g} \mathrm{g} \mathrm{OC}^{-1}$; conversely, the average sum of the brGDGTs in the sediments, $70 \pm 26 \mu \mathrm{g} \mathrm{OC}^{-1}$, is the largest out of the entire transect (Fig. 3a-b; Table 3). The BIT index is fairly high and constant throughout the sediment core, with an average value of $0.9 \pm 0.0$ (Fig. 3c; Table 3). The distribution of brGDGTs (Fig. 5d) is somewhat similar to that of the riverine SPM (Fig. 5c) and shows no major changes over the Holocene. The Tagus River Floodplain sediments has the lowest average values for MBT' 5 me $(0.4 \pm 0.1)$ and IR $(0.4 \pm 0.0)$ of all the sediment records in the transect (Fig. 3e-f). The mean DC' throughout the sediments in this sample set is $0.4 \pm 0.1$ (Fig. 3d; Table 3). 
(a)

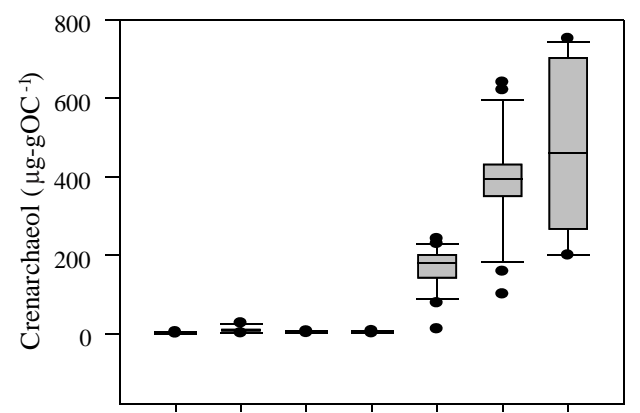

(b)

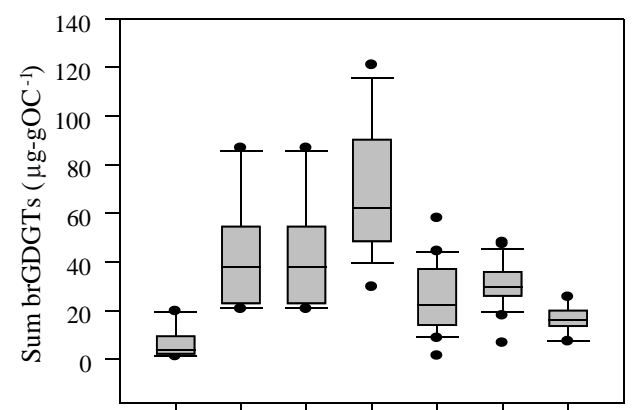

(c)

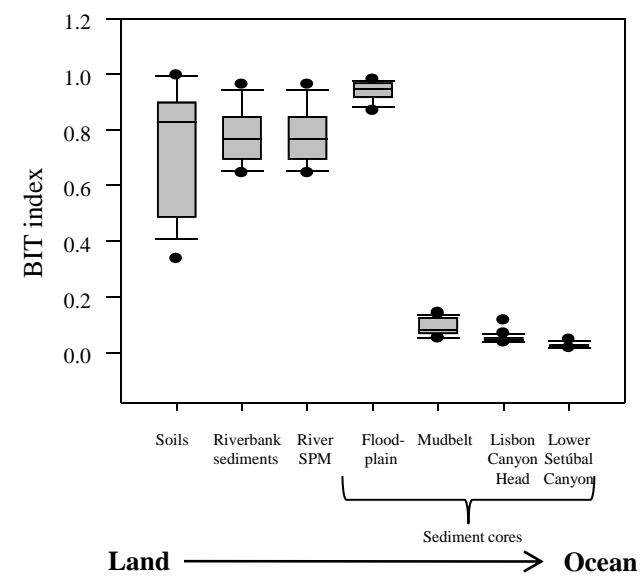

(d)

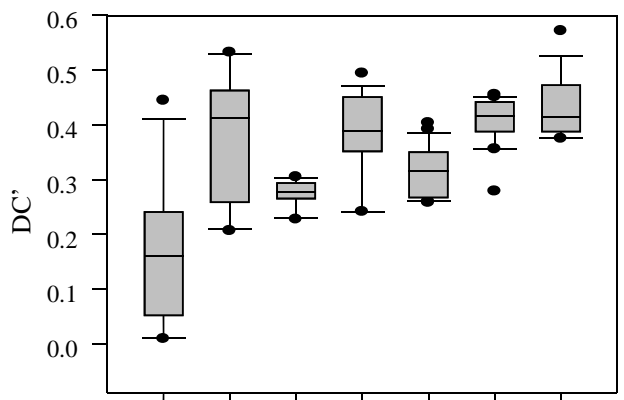

(e)

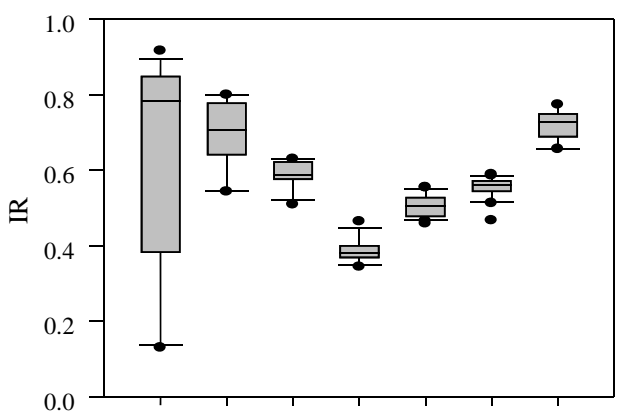

(f)

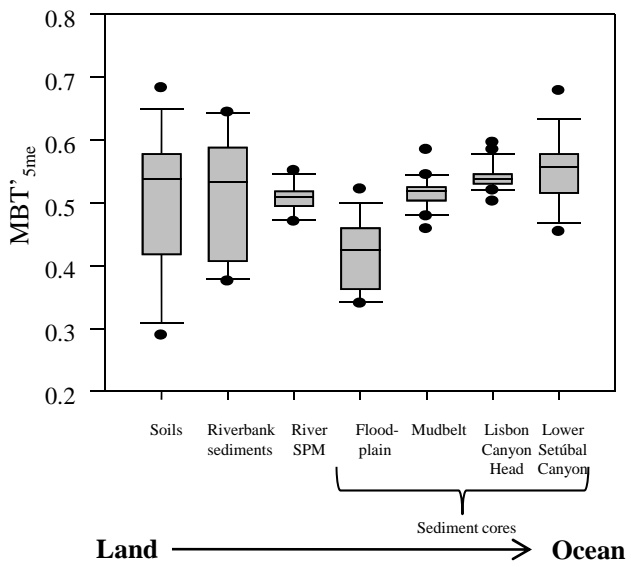

Figure 3. Box plots of (a) crenarchaeol concentrations $\left(\mu \mathrm{gg} \mathrm{OC}{ }^{-1}\right)$, (b) sum of brGDGTs $\left(\mu \mathrm{gg} \mathrm{OC}{ }^{-1}\right)$, (c) BIT index, (d) DC', (e) IR, and (f) $\mathrm{MBT}^{\prime}{ }_{5 \mathrm{me}}$ for each sample set in the transect from the land to the ocean off the Portuguese coast.

\subsubsection{Tagus Mudbelt sediments}

The average concentration of the brGDGTs in the Tagus Mudbelt sediments, $25 \pm 14 \mu \mathrm{g} \mathrm{g} \mathrm{OC}-1$, is lower than in the Tagus River Floodplain sediments; however, the concentration of crenarchaeol, $170 \pm 50 \mu \mathrm{g} \mathrm{g} \mathrm{OC}-1$, is higher in the Tagus Mudbelt sediments (Fig. 3a-b; Table 3). This results in a lower mean value of the BIT index (i.e., $0.09 \pm 0.03$; Fig. 3c; Table 3). The brGDGT distribution is relatively constant over the Holocene and is fairly similar to that of the Tagus River Floodplain sediments, with slightly higher fractional abundances of Ia and IIIa' (cf. Fig. 5d-e; Table 3). The average value of the MBT' 5 me $(0.5 \pm 0.0)$ is similar to the Tagus River SPM value (Fig. 3f). The average value of the
$\mathrm{DC}^{\prime}$ is $0.3 \pm 0.1$ and the mean value of the IR is $0.5 \pm 0.0$ (Fig. 3d-e; Table 3).

\subsubsection{Lisbon Canyon Head sediments}

The average sum of the brGDGTs, $31 \pm 9.3 \mu \mathrm{g} \mathrm{OC}^{-1}$, is about the same in the Lisbon Canyon Head sediments as in the Tagus Mudbelt sediments, but the amount of crenarchaeol, $390 \pm 130 \mu \mathrm{g} \mathrm{OC}^{-1}$, is larger in the Lisbon Canyon Head sediments (Fig. 3a-b; Table 3). This results in lower BIT index values $(0.05 \pm 0.02)$ than in the Tagus Mudbelt sediments (Fig. 3c; Table 3). The average brGDGT distribution (Fig. 5f) is fairly similar to that of the Tagus River Floodplain and Tagus Mudbelt sediments and is relatively constant over the Holocene. The average of the MBT' ${ }_{5 \mathrm{me}}(0.5 \pm 0.0)$ is statistically identical to that in the Tagus Mudbelt sedi- 


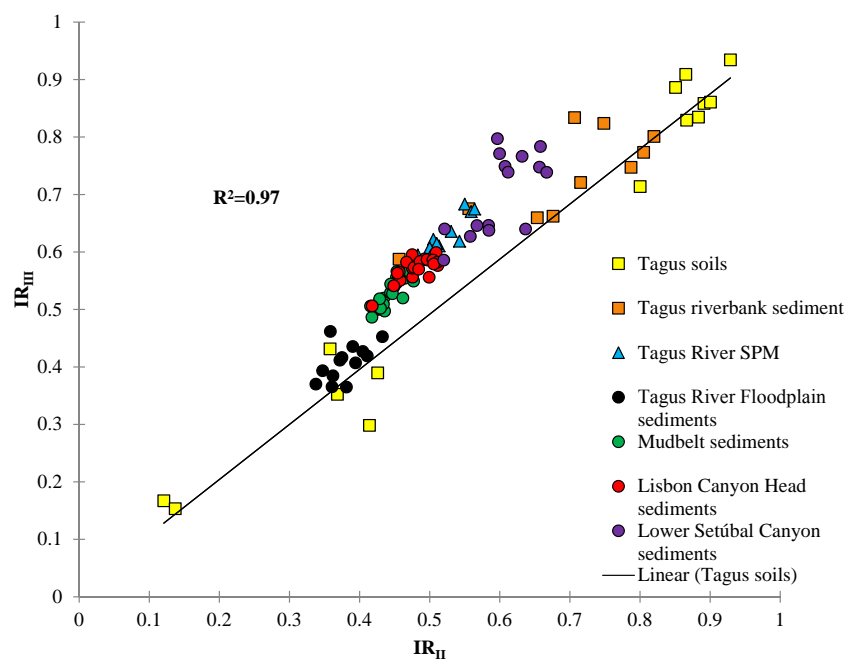

Figure 4. Isomer ratio for the non-cyclized pentamethylated

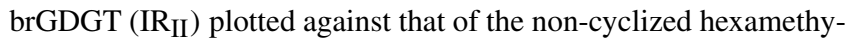
lated brGDGT ( $\left.\mathrm{IR}_{\mathrm{III}}\right)$.

ments (Fig. 3f; Table 3). However, the average IR $(0.6 \pm 0.0)$ and DC' $(0.4 \pm 0.0)$ values are both a bit higher (Fig. 3d-e; Table 3).

\subsubsection{Lower Setúbal Canyon sediments}

The concentrations of the brGDGTs in these most distal sediments are quite low, on average $16 \pm 5.5 \mu \mathrm{g} \mathrm{OC}-1$ (Fig. 3a; Table 3), while the amount of crenarchaeol in this sediment core is the highest out of the entire transect at $470 \pm 200 \mu \mathrm{g} \mathrm{OC}^{-1}$ (Fig. 3b; Table 3). This results in a low average BIT index value of $0.02 \pm 0.01$ (Fig. 3c; Table 3). The average distribution of brGDGTs in these sediments (Fig. $5 \mathrm{~g}$ ) is different from the marine sediments from the other two sites, with a higher fractional abundance of IIIa'. However, another component with the same molecular ion eluted at around the same time as IIIa' in the Lower Setúbal Canyon sediments (which we determined was not the "mixed 5,6-dimethyl isomer"; cf. Weber et al., 2015), complicating integration and quantification. This indicates that the brGDGT results from these sediments must be interpreted with some caution. The average MBT' ${ }_{5 \mathrm{me}}(0.6 \pm 0.1)$ and DC' $(0.4 \pm 0.1)$ values are fairly similar to the Lisbon Canyon Head sediments averages, but the average IR $(0.7 \pm 0.0)$ is the highest of all sediments (Fig. 3d-f; Table 3 ).

\subsection{PCA}

In order to determine the variation in the distribution of brGDGTs, we performed PCA on the distributions of brGDGTs of all the samples examined. Most variation is explained by principal component 1 (PC1; 29.8\%) and is clearly related to the fractional abundance of the 5-methyl vs. 6-methyl brGDGTs (Fig. 6a). With the exception of IIIc

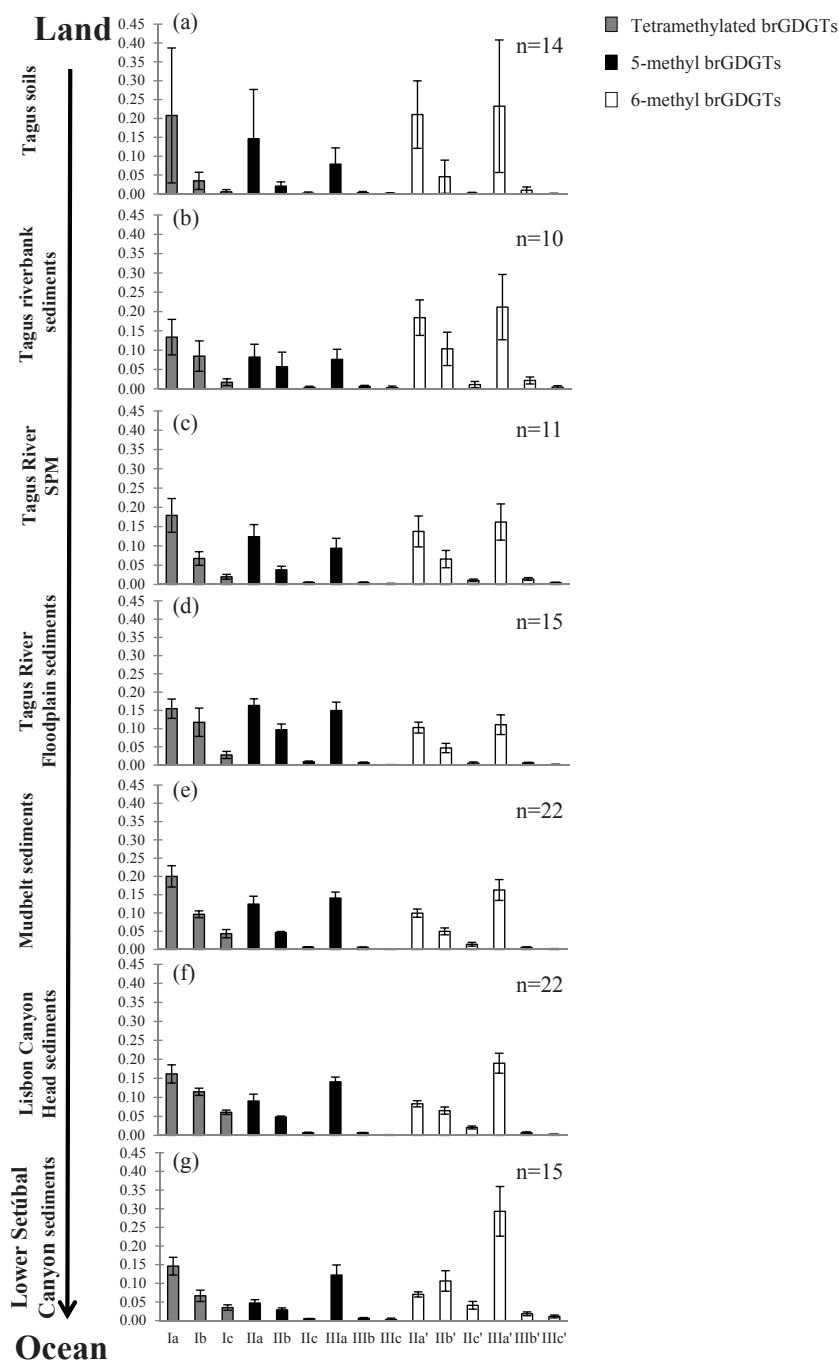

Figure 5. Average distribution of brGDGTs for each sample set along the transect of samples that runs from the land to the ocean off the coast of Lisbon. Evident from this figure is that the distribution of brGDGTs within this sample set varies greatly. Distributions of brGDGTs in marine sediments only reflect the distribution of the brGDGTs from the Tagus soils to a minor extent. The color of the bars reflects the brGDGT structure as labeled in the legend, and the range indicated with the error bars equals $2 x$ s the standard deviation.

(which is typically a minor brGDGT with a fractional abundance of $<1 \%$; Fig. 5), all of the 5-methyl brGDGTs score positively on PC1 and the 6-methyl brGDGTs score negatively. For the overall dataset, $\mathrm{PC} 1$ is highly negatively correlated with the IR ratio (Fig. $7 \mathrm{a}, R^{2}=0.78$ ). PC2 explains $25.6 \%$ of the variance of the PCA. Branched GDGTs that score positively on PC2 are generally comprised of cyclized and more methylated brGDGTs (Fig. 6a). With the exception of IIIc (which is typically a minor brGDGT with a fractional abundance of $<1 \%$; Fig. 4 ), all of the tetra- and pentamethylated brGDGTs containing no cyclopentane moiety 

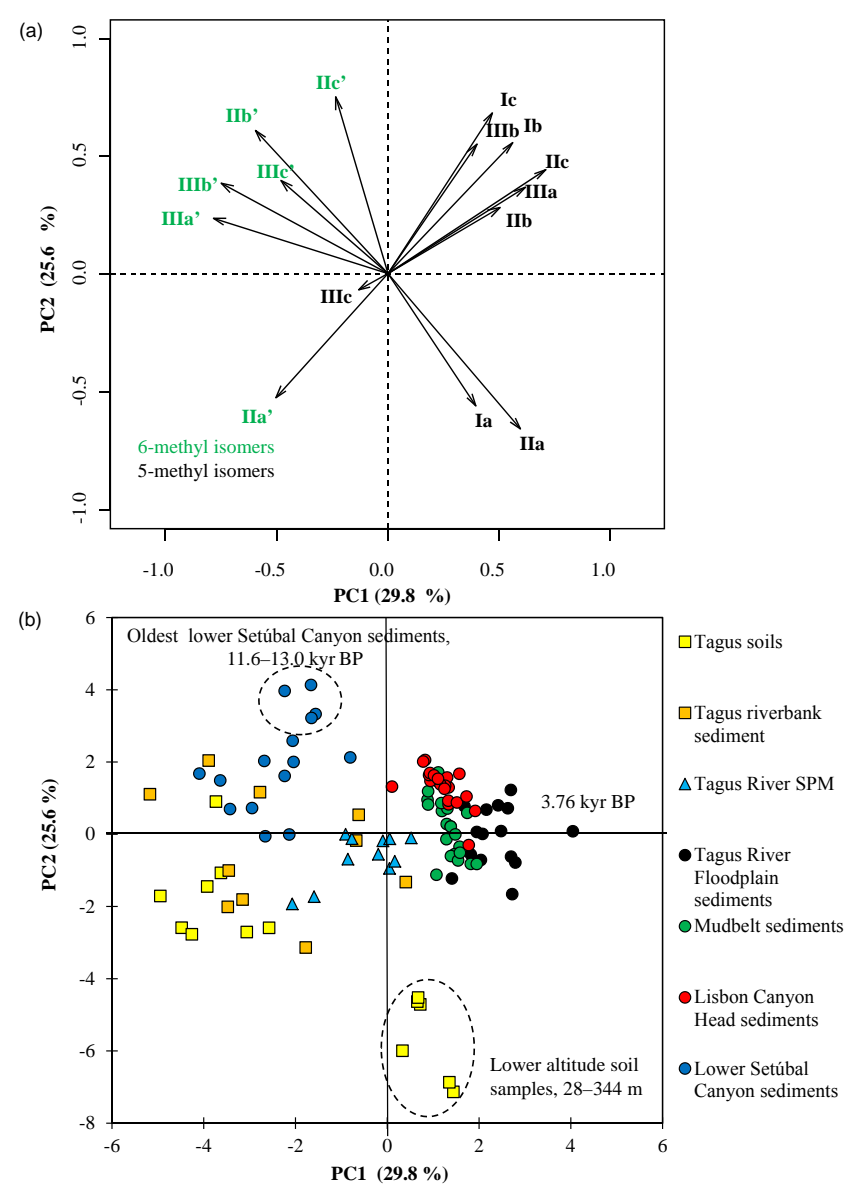

Figure 6. Principal component analysis based on the fractional abundances of the 15 brGDGTs of samples in the transect that runs from inland to off the coast of Portugal plotting (a) the scores of the brGDGT compounds on the first two principal components and (b) the scores of the samples from each sample set used in this study.

(i.e., Ia, IIa, and IIa') score negatively on PC2. Consequently, PC2 is highly positively correlated with DC' for the whole dataset (Fig. $7 \mathrm{~b}, R^{2}=0.84$ ).

\section{Discussion}

\subsection{Environmental parameters affecting brGDGT distribution in Tagus soils}

What is evident from the earlier study by Zell et al. (2014) was that the distribution of the brGDGTs in Tagus soils varies widely. The primary environmental parameters influencing brGDGT distributions in soil (Weijers et al., 2006), i.e., MAT and $\mathrm{pH}$, differed substantially in the Tagus River basin. MAT varies from 10 to $17^{\circ} \mathrm{C}$ and $\mathrm{pH}$ from 5.5 to 8.6 (Zell et al., 2014) and both parameters show a distinct correlation with altitude $\left(R^{2}=0.93\right.$ and 0.73 , respectively). Applying the brGDGT global soil calibration of Peterse et al. (2012), Zell et al. (2014) arrived at unrealistically low $\left(0-10^{\circ} \mathrm{C}\right)$ estimated MATs using the brGDGT distributions. This was attributed to the arid conditions in the region (MAP $<800 \mathrm{~mm} \mathrm{yr}^{-1}$ ), which have in other studies, including one that analyzed soils from the Iberian Peninsula, been indicated as a likely cause of the discrepancy between actual and reconstructed MAT using brGDGT distributions (Peterse et al., 2012; Dirghangi et al., 2013; Menges et al., 2014). Our reanalysis of the soils taking into account the novel 6-methyl brGDGTs now provides the possibility to reevaluate these data. It is clear that the fractional abundances of the novel 6-methyl brGDGTs vary to a large extent. The $\mathrm{IR}_{\mathrm{II}}$ and $\mathrm{IR}_{\mathrm{III}}$ vary from 0.1 to 0.9 (Fig. 4 ) and some of the soils score very negatively on PC1 (Fig. 6b), which is predominantly determined by the fractional abundance of the 6methyl brGDGTs. From the global soil brGDGT dataset (De Jonge et al., 2014a) it was evident that the main factor influencing the fractional abundance of the 6-methyl brGDGTs is soil $\mathrm{pH}$, with an increased abundance in high $\mathrm{pH}$ soils. In the Tagus River basin soil $\mathrm{pH}$ indeed shows a large variation, i.e., from 5.5 to 8.6, and this likely explains the large variation in IR. When we calculate the $\mathrm{pH}$ from the brGDGT distribution using the new Eq. (9) of De Jonge et al. (2014a), which is based predominantly on the fractional abundances of 6methyl brGDGTs, we find a highly significant correlation between measured and reconstructed $\mathrm{pH}\left(R^{2}=0.89\right)$ following the 1:1 line (Fig. 8a). Differences in soil pH also affect the degree of cyclization of brGDGTs (Weijers et al., 2007a; De Jonge et al., 2014b), and indeed we find a significant positive correlation between DC' and soil $\mathrm{pH}\left(R^{2}=0.74\right)$. The effect of MAT is not clearly revealed in the dataset. For the global soil brGDGT dataset a strong relationship exists between MAT and MBT' ${ }_{5 \mathrm{Me}}$ (De Jonge et al., 2014a). Although we observe substantial variation for MBT' ${ }_{5 \mathrm{Me}}$ in soils (i.e., $0.3-0.7$; Fig. 3f) for this dataset, we do not observe a statistically significant relationship of MAT with MBT' ${ }_{5 \mathrm{Me}}$. Also, reconstructed MATs are far too low, i.e., $0.5-13^{\circ} \mathrm{C}$ using Eq. (6) and $2.6-11^{\circ} \mathrm{C}$ using Eq. (10). Evidently, the "cold bias" of the brGDGT distributions in the soils of the Tagus river basin (Zell et al., 2014) is not solved when 5- and 6methyl brGDGTs are individually quantified.

Previously it was postulated that, in this region, aquatic in situ production and arid conditions are complicating the use of brGDGTs for climate reconstructions (Menges et al., 2014; Zell et al., 2014). Within the soil sample set, a strong negative relationship exists between the DC' and the measured MAT in the Tagus basin $\left(R^{2}=0.79\right)$, whereas the degree of cyclization up until this point has only been reported to be related to $\mathrm{pH}$ and not to MAT (Weijers et al., 2007a). Conversely, though, the $\mathrm{MAT}_{\mathrm{mrs}}$ reconstructed values for the soils have a positive correlation with DC' $\left(R^{2}=0.51\right)$ and it is lower than with the measured MAT. Although at this point we are unsure whether this association occurs in other arid areas as well, we do believe this strong relationship between the DC' and the MAT could be affecting the applicability of brGDGTs for temperature reconstructions in this region. 

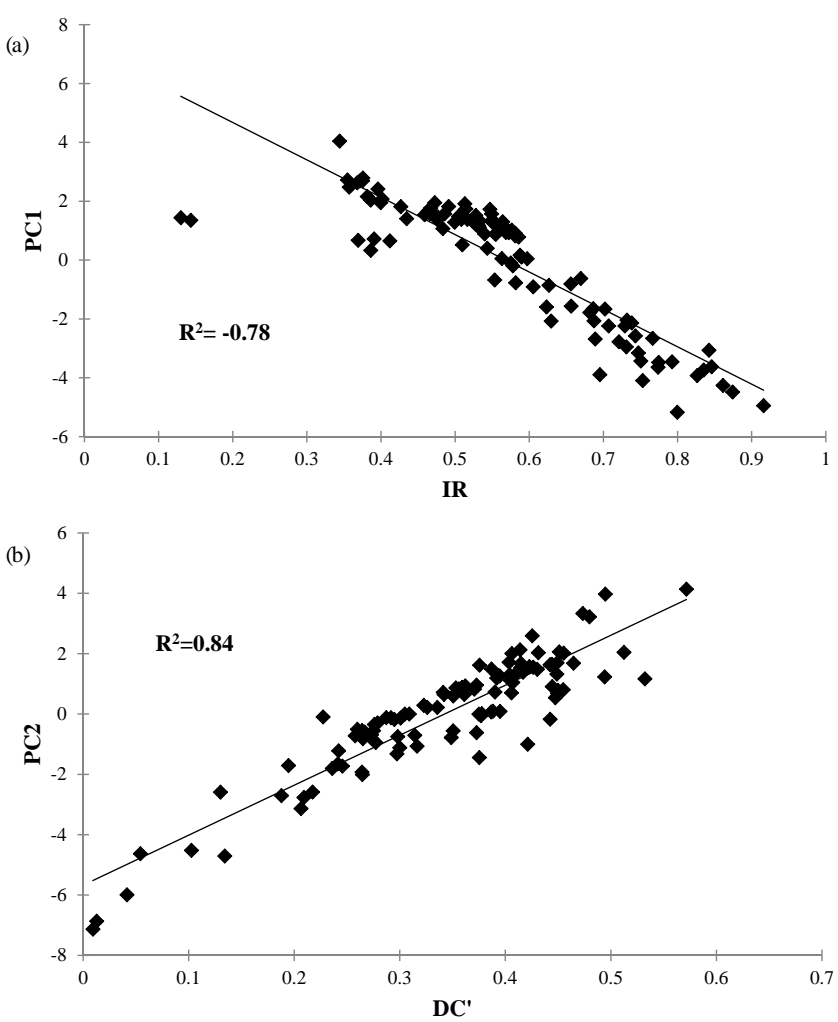

Figure 7. Scatterplots of (a) PC1 against the IR $\left(R^{2}=0.78\right)$ and (b) PC2 against DC' $\left(R^{2}=0.84\right)$ for the entire set of samples used in this study.

\subsection{Provenance of brGDGTs in the Tagus River and its outflow}

The application of brGDGTs in marine sediments influenced by river outflows for reconstruction of the continental paleoclimate (e.g., Weijers et al., 2006) rests on the premise that the distribution of the brGDGTs produced in the soils must be conserved throughout riverine transport to the sediments where they are archived. Therefore, we compare brGDGT distributions and concentrations from the rest of the sample set in the source-to-sink transect to determine whether the soil signal is conserved during transport in the Tagus River basin. The PCA results (Fig. 6b) indicate that for the most part the distribution of brGDGTs from the river SPM and sediments along the transect is not similar to those from the soils or the Tagus Watershed. Sediments from three of the sample sets in the transect - the Tagus River Floodplain sediments, the Tagus Mudbelt sediments, and the Lisbon Canyon Head sediments - all plot differently from the soils, and although the distributions of the Lower Setúbal Canyon sediments and the Tagus River SPM plot closer, there is still an offset from the soils. The Tagus riverbank sediments plot the most closely to the soil samples in the Tagus River basin; however, again a slight offset still exists. Thus, even without considering the effects of environmental param- eters on brGDGT distributions, we can already conclude that the brGDGTs in the sediments and river SPM only reflect the distribution of brGDGTs in the Tagus soils to a minor extent, and thus it is unlikely that Tagus soils are a major source for brGDGTs in the marine sediments.

Using PCA (Fig. 6) we tried to determine what factors are causing the variation in the distribution of brGDGTs in the Tagus River basin. PC1 is primarily related to the predominance of 5-methyl vs. 6-methyl brGDGTs (Fig. 6a) and thus pH (cf. De Jonge et al., 2014a). This was confirmed for the soils in the Tagus Basin, where the calculated $\mathrm{pH}$ based on the fractional abundance of predominantly 6methyl brGDGTs shows good agreement with the measured $\mathrm{pH}$ (see Sect. 4.1). De Jonge et al. (2014b) showed that, in the SPM of the alkaline waters of the river Yenisei, 6methyl brGDGTs also predominate, indicating that $\mathrm{pH}$ in all kinds of environmental settings determines the ratio between 5 and 6-methyl brGDGTs. The Tagus riverbank sediments, river SPM, and the Lower Setúbal Canyon sediments score mostly negatively on PC1, as do soils from higher altitudes (>350 m; Fig. 6b). The Tagus Mudbelt sediments, Lisbon Canyon Head sediments, the Tagus River Floodplain sediments, and the lower-altitude soils $(<350 \mathrm{~m})$ have similar abundances of the 5- and 6-methyl brGDGTs or higher abundances of the 5-methyl brGDGTs and plot mostly positively on PC1. Since the Tagus River Floodplain sediments, the Tagus Mudbelt sediments, and the Lisbon Canyon Head sediments do not have a predominance of 6-methyl brGDGTs, this indicates either that they received an equal contribution of soil-derived organic matter from the lower-altitude soils in the region $(<350 \mathrm{~m})$ as from the higher-altitude region $(>350 \mathrm{~m})$ or, more likely, that in situ production of brGDGTs is a large source of brGDGTs in these sample sets.

PC2 also explains a substantial part of the variance in the dataset $(25.6 \%$, Fig. $6 \mathrm{~b})$ and is correlated with DC' $\left(R^{2}=0.84, n=109\right.$, Fig. $\left.7 \mathrm{~b}\right)$. Since $\mathrm{pH}$ is also the main driver of DC' (Weijers et al., 2007a), this suggests that differences in $\mathrm{pH}$ are also responsible for the variance seen in PC2. The samples that stand out are the sediments from the Lower Setúbal Canyon core, which are the most marine sediments in the sample set and plot most positively, and the lowest-altitude soils (28-344 m), which plot the most negatively. These latter soils are characterized by a low measured $\mathrm{pH}$. The oldest (11.6-13.0 kyr BP) sediments of the Lower Setúbal Canyon score most positively on PC2. A high degree of cyclization of brGDGTs has been observed previously in marine sediments from a Svalbard fjord and attributed to marine in situ production in the alkaline pore waters of marine sediments (Peterse et al., 2009; Weijers et al., 2014). Reanalysis of the Svalbard sediments for brGDGTs actually showed that this cyclization affects the tetra- and pentamethylated brGDGTs to a much larger extent than that of the hexamethylated brGDGTs (Sinninghe Damsté, 2016), and the same observation can be made for the sediments of the Lower Setúbal Canyon (Fig. 5g). Evidently, the high degree of cyclization 

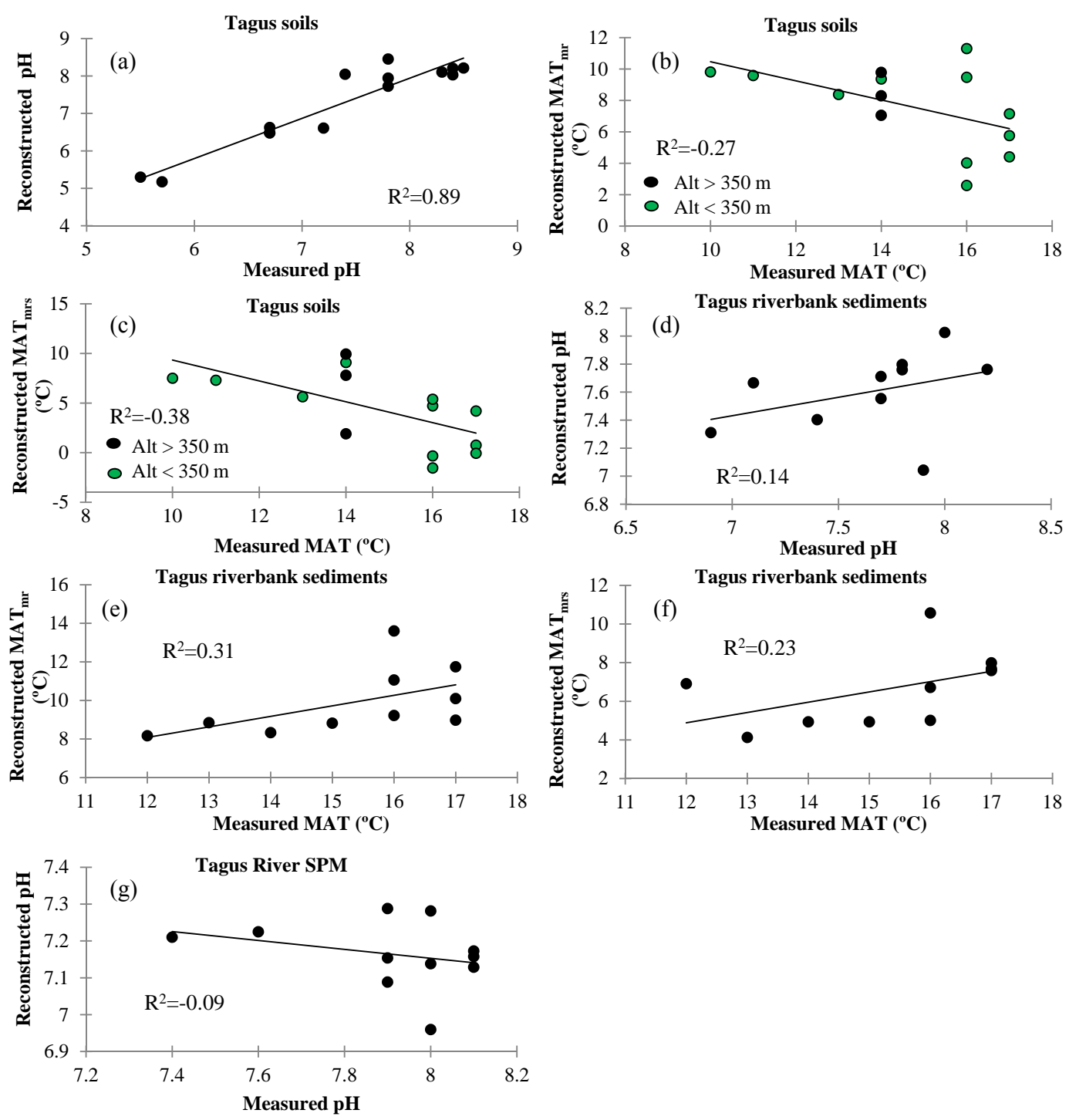

Figure 8. Panels (a)-(c) show scatterplots of the Tagus soil samples for (a) reconstructed and measured $\mathrm{pH}\left(R^{2}=0.89\right)$, (b) reconstructed MAT $_{\mathrm{mr}}\left({ }^{\circ} \mathrm{C}\right)$ and measured MAT $\left({ }^{\circ} \mathrm{C} ; R^{2}=0.27\right)$, and (c) reconstructed MAT $\mathrm{Mrs}\left({ }^{\circ} \mathrm{C}\right)$ and measured MAT $\left({ }^{\circ} \mathrm{C} ; R^{2}=0.38\right)$. For panels $(\mathbf{b})-$ (c) the soil samples from an altitude greater than $350 \mathrm{~m}$ are indicated in black and those from an altitude below $350 \mathrm{~m}$ are indicated in green. Panels (d)-(f) show scatterplots of the Tagus riverbank sediments for (d) reconstructed and measured $\mathrm{pH}\left(R^{2}=0.14\right)$, (e) reconstructed $\mathrm{MAT}_{\mathrm{mr}}\left({ }^{\circ} \mathrm{C}\right)$ and measured MAT $\left({ }^{\circ} \mathrm{C} ; R^{2}=0.31\right)$, and (f) reconstructed MAT $\operatorname{mrs}\left({ }^{\circ} \mathrm{C}\right)$ and measured MAT $\left({ }^{\circ} \mathrm{C} ; R^{2}=0.23\right)$. Panel $(\mathbf{g})$ is a scatterplot showing the reconstructed and measured $\mathrm{pH}$ for the Tagus River SPM samples $\left(R^{2}=0.09\right)$.

of brGDGTs as a response to $\mathrm{pH}$ is not as clearly seen in the soils since the high-altitude, high-pH soils from the Tagus watershed (Fig. S3c) do not exhibit the pattern (i.e., fractional abundance of IIb' larger than that of IIa') observed in the Lower Setúbal Canyon sediments (Fig. $5 \mathrm{~g}$ ). This pattern is, to a lesser degree, also seen in the sediments of the Lisbon Canyon Head core (Fig. 5f). As mentioned earlier, the Lower Setúbal Canyon sediments also display a predominance of 6methyl brGDGTs over the 5-methyl counterparts, especially with regard to the hexamethylated brGDGTs. In the Lower Setúbal Canyon sediments, IIIa' is by far the most abundant brGDGT, consisting of $29 \%$ of the entire brGDGT pool
(Fig. 5g). This is comparable to Svalbard sediments (Sinninghe Damsté, 2016), where IIIa' is also the most abundant brGDGT. Taken together this clearly indicates the influence of in situ production in the Lower Setúbal Canyon sediments. However, the degree of cyclization for Ia-c and IIa-c is not as high as observed for the Svalbard sediments, which still suggests some allochthonous input of brGDGTs even in these remote marine sediments.

Another way to determine whether in situ production is a factor affecting the brGDGT distribution in aquatic environments is by the calculation of reconstructed $\mathrm{pH}$ values. If in situ production is heavily contributing to the brGDGT 
pool, then the reconstructed $\mathrm{pH}$ values should reflect that of the aquatic environment in which they were produced. The average reconstructed $\mathrm{pH}$ of the sample sets in the transect is relatively high with a clear trend toward higher values with increasing distance from the river mouth (Fig. 9a), which would be in line with increased in situ production of brGDGTs in the alkaline pore waters of marine sediments. However, these values are still within the range of the measured (5.5-8.5) and reconstructed (Fig. 9a) pH of the soils, and so this does not prove in situ production as a major contributor of brGDGTs in these sample sets. Conversely, the newly calculated DC', also a reflection of $\mathrm{pH}$, is quite variable throughout the sample sets in the transect except for in the river SPM, where it is fairly constant (Fig. 3d; Table 3). Since the DC' is lowest in the soils $(0.2 \pm 0.1)$ and then higher in the rest of the samples in the transect $(0.3-$ 0.4 ), this suggests in situ production is an issue (cf. Zell et al., 2014) in all of the sample sets (Fig. 3d; Table 3).

\subsection{Branched GDGTs as indicators of terrestrial OM transport by the Tagus River}

Classically, the assessment of the contribution of terrestrial $\mathrm{OM}$ to marine sediments is performed by measuring $\delta^{13} \mathrm{C}_{\mathrm{TOC}}$ (Hedges and Oades, 1997, and references cited therein). In the earlier study of the Tagus River system, Zell et al. (2014) determined that the average $\delta^{13} \mathrm{C}_{\mathrm{TOC}}$ of the riverine SPM $(\sim-29 \pm 0.8 \%$ ) , like the Tagus soils, is consistent with a predominant $\mathrm{C}_{3}$ of higher plants origin (Fry and Sherr, 1984). Additionally, this study found the $\delta^{13} C_{\text {TOC }}$ in marine surface sediments off the Portuguese coast in front of the Tagus River increase with increasing distance offshore by an increased contribution of ${ }^{13} \mathrm{C}$-enriched marine OM. This trend is also evident for the Holocene sediments studied here. The most terrestrial sediments of the transect, i.e., from the Tagus River Floodplain, also have a $\delta^{13} \mathrm{C}_{\mathrm{TOC}}$ value $(\sim 27 \pm 1.0 \%$; Fig. 2 a; Table 3$)$ consistent with a predominant $\mathrm{C}_{\text {origin }}$ of higher plants. Moving offshore, the less negative $\delta^{13} \mathrm{C}_{\mathrm{TOC}}$ values of the Tagus Mudbelt sediments $(-24 \pm 0.2 \%$ ) , the Lisbon Canyon Head sediments $(-23 \pm 0.6 \%$ ) and the Lower Setúbal Canyon sediments $(-23 \pm 1.5 \%)$ all indicate that the majority of the TOC off the Portuguese shelf is of marine origin (Fig. 2a; Table 3). Therefore, as Zell et al. (2014) found with marine surface sediments off the Portuguese coast, the $\delta^{13} \mathrm{C}_{\mathrm{TOC}}(\%$ ) averages from the sediments in our transect also increase with increasing distance offshore, demonstrating that the present trend in the $\delta^{13} \mathrm{C}_{\mathrm{TOC}}$ signal remained the same over the Holocene.

Zell et al. (2014) previously showed that in the present-day Tagus River system the amount of brGDGTs ( $\mu \mathrm{g} \mathrm{g} \mathrm{OC}^{-1}$ ) increases from the soils to the riverbank sediment to the river SPM and explained this increase as proof of riverine in situ production of brGDGTs. Concentrations of summed brGDGTs in surface sediments in transects from the Por- (a)

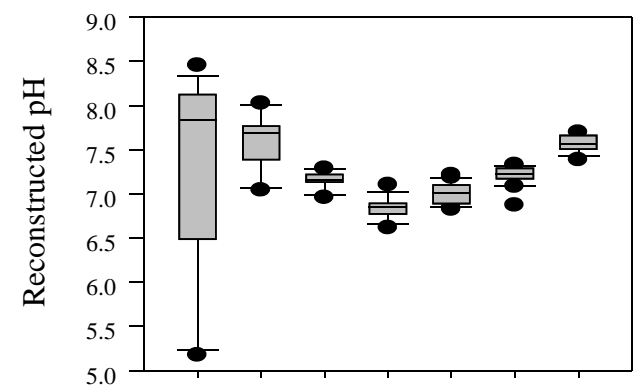

(b)

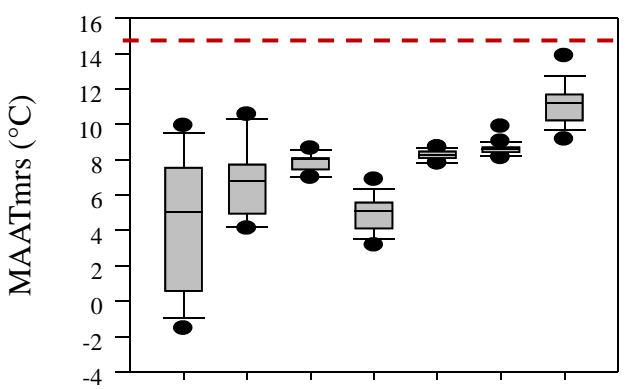

(c)

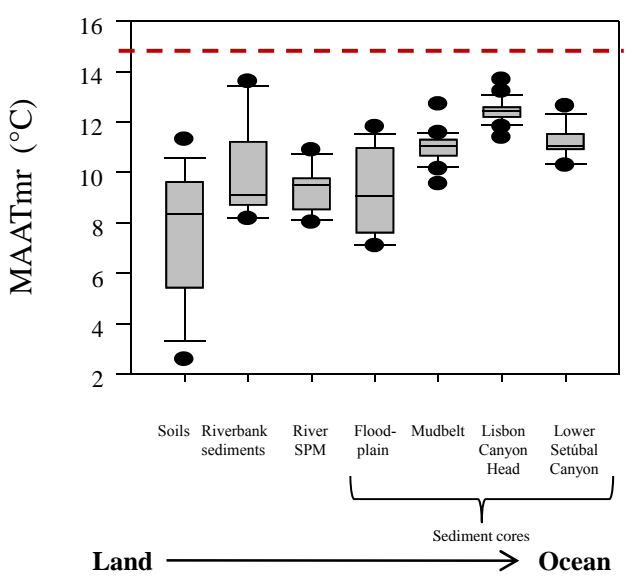

Figure 9. Box plots of all the sample sets within the transect from the land to the deep ocean off the Portuguese coast for (a) reconstructed $\mathrm{pH}$, (b) $\mathrm{MAT}_{\mathrm{mrs}}\left({ }^{\circ} \mathrm{C}\right)$, and (c) $\mathrm{MAT}_{\mathrm{mr}}\left({ }^{\circ} \mathrm{C}\right)$. Red dotted line indicates estimated present-day MAT for the Tagus River basin $\left(14.6^{\circ} \mathrm{C}\right)$.

tuguese coast rapidly declined with increasing distance from the coast, suggesting that brGDGTs could still be used as a tracer for terrestrial organic matter (Zell et al., 2015). The trends observed in these earlier studies are confirmed here for the Holocene. The Tagus River Floodplain sediments have the highest concentration of brGDGTs $\left(67 \pm 26 \mu \mathrm{g} \mathrm{OC}^{-1}\right)$ in the entire transect, much higher than in the soils (Fig. 3b; Table 3). However, the sediments in this core are somewhat atypical for the Tagus River Floodplain as some layers consist of peat as a result of the low-energy backswamp conditions in the vicinity, which could explain the difference in brGDGT concentrations from the surrounding soils. This could also be due to the addition of aquatically produced brGDGTs from the river during times of flooding, although 
it should be noted that the concentration of brGDGTs is even higher than in riverine SPM (Fig. 3b). The summed brGDGT concentration decreases and is fairly similar among the Tagus Mudbelt sediments $\left(25 \pm 14 \mu \mathrm{g} \mathrm{OC}^{-1}\right)$ and the Lisbon Canyon Head sediments $\left(31 \pm 9.3 \mu \mathrm{g} \mathrm{OC}^{-1}\right)$, and then decreases further moving away from the coastline to the Lower Setúbal Canyon sediments $\left(16 \pm 5.5 \mu \mathrm{gg} \mathrm{OC}{ }^{-1}\right)$ demonstrating the decrease in input of riverine brGDGTs moving away from the shoreline (Fig. 3b). However, even though the sum of the brGDGTs is lower in the marine sediment than in the Tagus River Floodplain sediments, the amount of brGDGTs in all four sediment cores is higher than in the Tagus soils $\left(\sim 6.8 \pm 6.5 \mu \mathrm{g} \mathrm{OC}^{-1}\right)$, indicating the origins of the brGDGTs in the sediment cores are not all soilderived and pointing instead to riverine in situ production as well as possibly in aquatic sediments (Fig. 3b).

A previous study by Zell et al. (2015) determined that, in the surface sediments off the coast of Portugal, the BIT index is influenced by both declining brGDGT concentrations and increased crenarchaeol production with increasing distance from the coast. For the Holocene sediments studied here, the average concentration of crenarchaeol in the Tagus River Floodplain sediments is low $\left(2.8 \pm 1.7 \mu \mathrm{g} \mathrm{OC}^{-1}\right)$ and similar to that of the Tagus soils $\left(1.4 \pm 1.1 \mu \mathrm{g} \mathrm{OC}^{-1}\right.$; Fig. 3a; Table 3). The crenarchaeol concentration increases in the sediments with increasing distance from the shoreline, signifying the increase in marine production with water depth and distance from the coast (Fig. 3a). Consequently, the BIT index is the highest in the Tagus River Floodplain sediments $(0.94 \pm 0.03)$ out of the entire transect (Fig. 3c), and then the BIT index decreases within the sediments along the transect with increasing distance from the Portuguese coast, potentially signifying a decrease in terrestrial input moving away from the shoreline.

\subsection{Factors affecting the application of brGDGTs for paleoclimate reconstructions off the Iberian Peninsula}

Despite the caveats with respect to in situ production of brGDGTs in aquatic environments as described in the previous section, we tested how the new soil calibration based on individually quantified 5-methyl and 6-methyl brGDGTs (De Jonge et al., 2014a) performed to reconstruct continental MAT in this region. For this comparison we will consider the present-day MAT of the entire Tagus River basin, $14.6 \pm 2.2^{\circ} \mathrm{C}$ (Zell et al., 2014), assuming that soil-derived brGDGTs from along the whole river basin are contributing to the marine sediments. The assumption that the brGDGTs from the entire Tagus River basin are being contributed to oceanic sediments is probably invalid for modern times as the construction of dams along the Tagus River, which began in the 1940s, most likely prevents part of the terrestrial material from upstream making it downstream and out off the coast of Portugal. However, since we are not look- ing at marine surface sediments in this study but instead sediments deposited during the Holocene, the placement of dams in the river should not affect our results except in the case of the riverine SPM. Despite the separation of the 5- and 6-methyl brGDGT isomers and the application of the new proxy, the reconstructed MATs using both riverine SPM and Holocene sediments is still substantially lower than $14.6^{\circ} \mathrm{C}$ (Fig. 9b and c), as noted for the Tagus Basin soils (see Sect. 4.1). Using the MAT $_{\mathrm{mr}}$ calibration, the reconstructed average temperature for the Lower Setúbal Canyon sediments $\left(11.2 \pm 0.7^{\circ} \mathrm{C}\right)$ is the most similar to modern-day MAT in the regions (Fig. 9b). Using the $\mathrm{MAT}_{\mathrm{mr}}$ calibration, the average reconstructed temperature of the Lisbon Canyon Head sediments, $12.4 \pm 0.5^{\circ} \mathrm{C}$, comes closest to the modernday MAT in the region (Fig. 9c).

Even though we used the new calibration to reconstruct MAT, it should be noted that the low BIT values $(<0.15$; Fig. 3c) of the Holocene sediments deposited at the three marine sites indicate that there were probably not enough soil-derived brGDGTs making it out to ocean and being deposited in the sediments over the Holocene for reliable climate reconstructions (cf. Weijers et al., 2014). When considering the summed concentration of brGDGTs along the entire transect, since the concentration is lowest in the soils, this indicates that the origin of the brGDGTs may not be solely soil-derived. Therefore, even though the BIT index seems high enough for MAT reconstructions in the riverbank sediments and river SPM, in situ production of brGDGTs could be complicating the applications of brGDGTs for paleoclimate reconstructions throughout the transect of samples as was previously discussed (see Sect. 4.2). This further supports earlier conclusions from previous studies (Yang et al., 2012; Zell et al., 2013) stating that the amount and origin of brGDGTs in a system need to be examined along with the BIT index when determining whether brGDGTs can be applied for MAT reconstruction.

\section{Conclusions}

We have established that the distribution of brGDGTs varies greatly within the Tagus River basin (Fig. 5), and although this may be partly explained by the varying contributions of higher altitude, which contain a greater proportion of 6methyl isomers, vs. lower-altitude soils in the sample sets, it is more likely due to the contribution of aquatically produced brGDGTs in some of the sample sets. In order to use sedimentary brGDGTs for paleoclimate reconstructions, the distribution of brGDGTs in the soils must be related to the MAT and conserved throughout riverine transport to the sediments where they are deposited; however, our results corroborate previous studies stating that most of the terrestrial matter is not making it out to the ocean and being deposited in sediments close to shore. The lack of soil-derived OM in offshore sediments along with the substantial input of aquatically pro- 
duced brGDGTs is complicating MAT reconstructions from sedimentary, marine brGDGTs in this region.

Additionally, we confirm the findings of Zell et al. (2014, 2015) that in situ production of brGDGTs is occurring in the river and marine systems of the Tagus River basin and go on to show that there are indications that it occurred in the past as well. Although in situ production is complicating environmental reconstructions using marine sediments, another issue is that accurate MAT reconstructions using brGDGTs cannot currently be performed on the soils, even with the separation of the 6-methyl brGDGTs from the 5-methyl isomers using the new method and calibrations. Previous studies have concluded that paleoclimate reconstructions in arid regions using brGDGTs are complicated due to a breakdown in the relationship with MBT' and MAT (Peterse et al., 2012; Menges et al., 2014). In this study we confirm that there is no strong relationship between the MBT' 5 me and measured MAT in this arid region. However, we also do not observe the same relationship with MAP and MBT' 5 me that has been previously reported between MAP and MBT' in arid regions and has been implied in making reconstructions difficult. Instead, we see a strong relationship with the DC' and measured MAT in the area not observed before. We also see a predominance of 6-methyl isomers, previously only reported in river SPM, in the Tagus soils from greater than $350 \mathrm{~m}$ altitude. Although this might be a characteristic of arid soils and related to MAP since it is below $550 \mathrm{~mm} \mathrm{yr}^{-1}$ in most of the soil samples above $350 \mathrm{~m}$, the two highest-elevation soil samples, which both have a MAP above $550 \mathrm{~mm} \mathrm{yr}^{-1}$, also demonstrate this trend. Future studies need to be performed in arid environments to determine whether a strong relationship between MAT and DC', as well as a predominance of 6-methyl isomers, is a characteristic of arid regions and contributing to the complications found using brGDGTs for paleoclimate reconstructions. Also, higher-elevation environments should be further studied to determine whether a predominance of 6-methyl brGDGTs is a feature of higher altitudes and complicating climate reconstructions.

Because of these unique features in this region, perhaps the development of a local calibration could assuage difficulties in using brGDGTs as a paleoclimate proxy for soils in the Tagus River basin. This would not, however, solve the issue of in situ-produced brGDGTs overwhelming the amount of soil-derived brGDGTs in aquatic sediments. We did find that the new CBT' and pH calibrations do an excellent job reconstructing $\mathrm{pH}$ in the soils of the Tagus Basin, and since $\mathrm{pH}$ is related to other environmental factors such as MAP, this will be useful for paleoclimate reconstructions in terrestrial sites over the Iberian Peninsula, where in situ production is not a complicating factor.

\section{Data availability}

The data presented in this paper can be found in Table 3 and S2.

\section{The Supplement related to this article is available online at doi:10.5194/bg-13-5719-2016-supplement.}

Author contributions. Jung-Hyun Kim and Jaap S. Sinninghe Damsté designed the study, which was carried out by Lisa Warden, who completed bulk carbon isotope and brGDGT analysis on samples along with Claudia Zell. Claudia Zell, Henko Stigter, Geert-Jan Vis, and Jung-Hyun Kim collected samples for this study. Jérôme Bonnin picked forams for dating. Lisa Warden and Jaap S. Sinninghe Damsté prepared the manuscript with contributions from all co-authors.

Acknowledgements. The research leading to these results has received funding from the European Research Council under the European Union's Seventh Framework Programme (FP7/20072013)/ERC grant agreement no. 226600. Jaap S. Sinninghe Damsté is supported by funding from the Netherlands Earth System Science Centre (NESSC) though a Gravitation grant from the Dutch Ministry for Education, Culture and Science. We thank the captain and crew of the RV Pelagia for their support with sampling. We thank J. Ossebaar and E. C. Hopmans for their help with the HPLC-APCI-MS analysis, J. Ossebaar for his help with the element analyzer, and L. A. C. Rosales and A. Mets for help with sample preparation.

Edited by: S. W. A. Naqvi

Reviewed by: R. Sparkes and one anonymous referee

\section{References}

Abrantes, F., Lebreiro, S., Rodrigues, T., Gil, I., Bartels-Jónsdóttir, H., Oliveira, P., Kissel, C., and Grimalt, J. O.: Shallow-marine sediment cores record climate variability and earthquake activity off Lisbon (Portugal) for the last 2000 years, Quaternary Sci. Rev., 24, 2477-2494, 2005.

Abrantes, F., Lopes, C., Rodrigues, T., Gil, I., Witt, L., Grimalt, J., and Harris, I.: Proxy calibration to instrumental data set: Implications for paleoceanographic reconstructions, Geochem. Geophy. Geosy., 10, Q09U07, doi:10.1029/2009GC002604, 2009.

Andreu, L., GutiéRrez, E., Macias, M., Ribas, M., Bosch, O., and Camarero, J. J.: Climate increases regional tree-growth variability in Iberian pine forests, Glob. Change Biol., 13, 1-12, 2007.

Bartels-Jónsdóttir, H. B., Knudsen, K. L., Abrantes, F., Lebreiro, S., and Eiríksson, J.: Climate variability during the last 2000 years in the Tagus Prodelta, western Iberian Margin: Benthic foraminifera and stable isotopes, Mar. Micropaleontol., 59, 83-103, 2006. 
Bartels-Jonsdottir, H. B., Voelker, A. H. L., Knudsen, K. L., and Abrantes, F.: Twentieth-century warming and hydrographical changes in the Tagus Prodelta, eastern North Atlantic, Holocene, 19, 369-380, 2009.

Bendle, J. A., Weijers, J. W., Maslin, M. A., Sinninghe Damsté, J. S., Schouten, S., Hopmans, E. C., Boot, C. S., and Pancost, R. D.: Major changes in glacial and Holocene terrestrial temperatures and sources of organic carbon recorded in the Amazon fan by tetraether lipids, Geochem. Geophy. Geosy., 11, Q12007, doi:10.1029/2010GC003308, 2010.

Benito, G., Díez-Herrero, A., and de Villalta, M. F.: Magnitude and frequency of flooding in the Tagus basin (Central Spain) over the last millennium, Climate Change, 58, 171-192, 2003.

Cachao, M. and Moita, M. T.: Coccolithus pelagicus, a productivity proxy related to moderate fronts off Western Iberia, Mar. Micropaleontol., 39, 131-155, 2000.

Corella, J. P., Stefanova, V., El Anjoumi, A., Rico, E., Giralt, S., Moreno, A., Plata-Montero, A., and Valero-Garcés, B. L.: A 2500-year multi-proxy reconstruction of climate change and human activities in northern Spain: The Lake Arreo record, Palaeogeogr. Palaeocl., 386, 555-568, 2013.

Davis, B. A. S., Brewer, S., Stevenson, A. C., and Guiot, J.: The temperature of Europe during the Holocene reconstructed from pollen data, Quaternary Sci. Rev., 22, 1701-1716, 2003.

De Jonge, C., Hopmans, E. C., Stadnitskaia, A., Rijpstra, W. I. C., Hofland, R., Tegelaar, E., and Sinninghe Damsté, J. S.: Identification of novel penta-and hexamethylated branched glycerol dialkyl glycerol tetraethers in peat using HPLC-MS ${ }^{2}$, GC-MS and GC-SMB-MS, Org. Geochem., 54, 78-82, 2013.

De Jonge, C., Hopmans, E. C., Zell, C. I., Kim, J.-H., Schouten, S., and Sinninghe Damsté, J. S.: Occurrence and abundance of 6-methyl branched glycerol dialkyl glycerol tetraethers in soils: Implications for palaeoclimate reconstruction, Geochim. Cosmochim. Ac., 141, 97-112, 2014a.

De Jonge, C., Stadnitskaia, A., Hopmans, E. C., Cherkashov, G., Fedotov, A., and Sinninghe Damsté, J. S.: In situ produced branched glycerol dialkyl glycerol tetraethers in suspended particulate matter from the Yenisei River, Eastern Siberia, Geochim. Cosmochim. Ac., 125, 476-491, 2014b.

De Jonge, C., Stadnitskaia, A., Hopmans, E. C., Cherkashov, G., Fedotov, A., Streletskaya, I. D., Vasiliev, A. A., and Sinninghe Damsté, J. S.: Drastic changes in the distribution of branched tetraether lipids in suspended matter and sediments from the Yenisei River and Kara Sea (Siberia): Implications for the use of brGDGT-based proxies in coastal marine sediments, Geochim. Cosmochim. Ac., 165, 200-225, 2015.

de Stigter, H. C., Jesus, C. C., Boer, W., Richter, T. O., Costa, A., and van Weering, T. C.: Recent sediment transport and deposition in the Lisbon-Setúbal and Cascais submarine canyons, Portuguese continental margin, Deep-Sea Res. Pt. II, 58, 2321-2344, 2011.

Dias, J. M. A., Jouanneau, J. M., Gonzalez, R., Araújo, M. F., Drago, T., Garcia, C., Oliveira, A., Rodrigues, A., Vitorino, J., and Weber, O.: Present day sedimentary processes on the northern Iberian shelf, Prog. Oceanogr., 52, 249-259, 2002.

Dirghangi, S. S., Pagani, M., Hren, M. T., and Tipple, B. J.: Distribution of glycerol dialkyl glycerol tetraethers in soils from two environmental transects in the USA, Org. Geochem., 59, 49-60, 2013.
Fletcher, W. J., Boski, T., and Moura, D.: Palynological evidence for environmental and climatic change in the lower Guadiana valley, Portugal, during the last 13000 years, Holocene, 17, 481-494, 2007.

Fry, B. and Sherr, E.: $\delta^{13} \mathrm{C}$ measurements as indicators of carbon flow in marine and freshwater ecosystems, Contrib. Mar. Sci., 27, 15-47, 1984.

Haug, G. H., Günther, D., Peterson, L. C., Sigman, D. M., Hughen, K. A., and Aeschlimann, B.: Climate and the collapse of Maya civilization, Science, 299, 1731-1735, 2003.

Hedges, J. I. and Oades, J. M.: Comparative organic geochemistries of soils and marine sediments, Org. Geochem., 27, 319-361, 1997.

Hopmans, E. C., Schouten, S., and Sinninghe Damsté, J. S.: The effect of improved chromatography on GDGT-based palaeoproxies, Org. Geochem., 93, 1-6, 2015.

Hren, M. T., Pagani, M., Erwin, D. M., and Brandon, M: Biomarker reconstruction of the early Eocene paleotopography and paleoclimate of the northern Sierra Nevada, Geology, 38, 7-10, 2010.

Huguet, C., Hopmans, E. C., Febo-Ayala, W., Thompson, D. H., Sinninghe Damsté, J. S., and Schouten, S.: An improved method to determine the absolute abundance of glycerol dibiphytanyl glycerol tetraether lipids, Org. Geochem., 37, 1036-1041, 2006.

Huntley, B. and Prentice, I. C.: July temperatures in Europe from pollen data, 6000 years before present, Science, 241, 687-690, 1988.

Hurrell, J. W.: Decadal trends in the North Atlantic oscillation, Science, 269, 676-679, 1995.

Hurrell, J. W. and Van Loon, H: Decadal variations in climate associated with the north Atlantic oscillation, Climate Change, 36, 301-326, 1997.

International Ocean Commission, International Hydrographic Organization, and British Oceanographic Data Centre (IOC/IHO/BODC): Centenary Edition of the GEBCO Digital Atlas, Published on CD-ROM on Behalf of the Intergovernmental Oceanographic Commission and the International Hydrographic Organization as part of the General Bathymetric Chart of the Oceans, edited by: British-Oceanographic-Data-Centre, Liverpool, United Kingdom, 2003.

Jarvis, A., Reuter, H. I., Nelson, A.,and Guevara, E.: Hole-filled seamless SRTM data vol. 3, International Centre for Tropical Agriculture (CIAT), 2006.

Jesus, C. C., de Stigter, H. C., Richter, T. O., Boer, W., Mil-Homens, M., Oliveira, A., and Rocha, F.: Trace metal enrichments in Portuguese submarine canyons and open slope: anthropogenic impact and links to sedimentary dynamics, Mar. Geol., 271, 72-83, 2010.

Jouanneau, J. M., Garcia, C., Oliveira, A., Rodrigues, A., Dias, J. A., and Weber, O.: Dispersal and deposition of suspended sediment on the shelf off the Tagus and Sado estuaries, SW Portugal, Prog. Oceanogr., 42, 233-257, 1998.

Keating-Bitonti, C. R., Ivany, L. C., Affek, H. P., Douglas, P., and Samson, S. D.: Warm, not super-hot, temperatures in the early Eocene subtropics, Geology, 39, 771-774, 2011.

Lastras, G., Arzola, R. G., Masson, D. G., Wynn, R. B., Huvenne, V. A. I., Hühnerbach, V., and Canals, M.: Geomorphology and sedimentary features in the Central Portuguese submarine canyons, Western Iberian margin, Geomorphology, 103, 310-329, 2009. 
Lebreiro, S. M., Francés, G., Abrantes, F. F. G., Diz, P., BartelsJónsdóttir, H. B., Stroynowski, Z. N., Gil, I. M., Pena, L. D., Rodrigues, T., and Jones, P. D.: Climate change and coastal hydrographic response along the Atlantic Iberian margin (Tagus Prodelta and Muros Ría) during the last two millennia, Holocene, 16, 1003-1015, 2006.

Le Pera, E. and Arribas, J.: Sand composition in an Iberian passivemargin fluvial course: the Tajo River, Sediment. Geol., 171, 261281, 2004.

Linan, I. D., Gutierrez, E., Andreu-Hayles, L., Heinrich, I., and Helle, G.: Potential to explain climate from tree rings in the south of the Iberian Peninsula, Clim. Res., 55, 119-134, 2012.

Martin-Chivelet, J., Belen Munoz-Garcia, M., Edwards, R. L., Turrero, M. J., and Ortega, A. I.: Land surface temperature changes in Northern Iberia since $4000 \mathrm{yr}$ BP, based on delta C-13 of speleothems, Global Planet. Change, 77, 1-12, 2011.

Menges, J., Huguet, C., Alcañiz, J. M., Fietz, S., Sachse, D., and Rosell-Melé, A.: Influence of water availability in the distributions of branched glycerol dialkyl glycerol tetraether in soils of the Iberian Peninsula, Biogeosciences, 11, 2571-2581, doi:10.5194/bg-11-2571-2014, 2014.

Munoz-Garcia, M. B., Martin-Chivelet, J., Rossi, C., Ford, D. C., and Schwarcz, H. P.: Chronology of termination II and the Last Interglacial Period in North Spain based on stable isotope records of stalagmites from Cueva del Cobre (Palencia), J. Iber. Geol., 33, 17-30, 2007.

Palumbo, E., Flores, J. A., Perugia, C., Emanuele, D., Petrillo, Z., Rodrigues, T., Voelker, A. H., and Amore, F. O.: Abrupt variability of the last $24 \mathrm{ka}$ BP recorded by coccolithophore assemblages off the Iberian Margin (core MD03-2699), J. Quaternary Sci., 28, 320-328, 2013.

Pancost, R. D. and Sinninghe Damsté, J.S.: Carbon isotopic compositions of prokaryotic lipids as tracers of carbon cycling in diverse settings, Chem. Geol., 195, 29-58, 2003.

Peterse, F., Kim, J.-H., Schouten, S., Kristensen, D. K., Kocc, N., and Sinninghe Damsté, J. S.: Constraints on the application of the MBT/CBT palaeothermometer at high latitude environments (Svalbard, Norway), Org. Geochem., 40, 692-699, 2009.

Peterse, F., Meer, J. van der, Schouten, S., Weijers, J. W., Fierer, N., Jackson, R. B., Kim, J.-H., and Sinninghe Damsté, J. S.: Revised calibration of the MBT-CBT paleotemperature proxy based on branched tetraether membrane lipids in surface soils, Geochim. Cosmochim. Ac., 96, 215-229, 2012.

Reimer, P. J., Bard, E., Bayliss, A., Beck, J. W., Blackwell, P. G., Ramsey, C. B., Buck, C. E., Cheng, H., Edwards, R. L., Friedrich, M., and Grootes, P. M.: IntCal13 and Marine13 radiocarbon age calibration curves 0-50 000 years cal BP, Radiocarbon, 55, 1869-1887, 2013.

Rodó, X., Baert, E., and Comín, F. A.: Variations in seasonal rainfall in Southern Europe during the present century: relationships with the North Atlantic Oscillation and the El Niño-Southern Oscillation, Clim. Dynam., 13, 275-284, 1997.

Rodrigues, T., Grimalt, J. O., Abrantes, F. G., Flores, J. A., and Lebreiro, S. M.: Holocene interdependences of changes in sea surface temperature, productivity, and fluvial inputs in the Iberian continental shelf (Tagus mud patch), Geochem. Geophy. Geosy., 10, Q07U06, doi:10.1029/2008GC002367, 2009.

Schouten, S., Eldrett, J., Greenwood, D. R., Harding, I., Baas, M., and Sinninghe Damsté, J. S.: Onset of long-term cooling of
Greenland near the Eocene-Oligocene boundary as revealed by branched tetraether lipids, Geology, 36, 147-150, 2008.

Sinninghe Damsté, J. S., Schouten, S., Hopmans, E. C., van Duin, A. C., and Geenevasen, J. A.: Crenarchaeol the characteristic core glycerol dibiphytanyl glycerol tetraether membrane lipid of cosmopolitan pelagic crenarchaeota, J. Lipid Res., 43, 16411651, 2002.

Sinninghe Damsté, J. S., Ossebaar, J., Abbas, B., Schouten, S., and Verschuren, D.: Fluxes and distribution of tetraether lipids in an equatorial African lake: Constraints on the application of the TEX $_{86}$ palaeothermometer and BIT index in lacustrine settings, Geochim. Cosmochim. Ac., 73, 4232-4249, 2009.

Sinninghe Damsté, J. S., Rijpstra, W. I. C., Hopmans, E. C., Weijers, J. W. H., Foesel, B. U., Overmann, J., and Dedysh, S. N.: 13,16Dimethyl Octacosanedioic Acid (iso-Diabolic Acid), a Common Membrane-Spanning Lipid of Acidobacteria Subdivisions 1 and 3, Appl. Environ. Microbiol., 77, 4147-4154, 2011.

Sinninghe Damsté, J. S., Rijpstra, W. I. C., Hopmans, E. C., Foesel, B. U., Wüst, P. K., Overmann, J., Tank, M., Bryant, D. A., Dunfield, P. F., Houghton, K., and Stott, M. B.: Ether- and EsterBound iso-Diabolic Acid and Other Lipids in Members of Acidobacteria Subdivision 4, Appl. Environ. Microbiol., 80, 52075218, 2014.

Sinninghe Damsté, J. S.: Spatial heterogeneity of sources of branched tetraethers in shelf systems: The geochemistry of tetraethers in the Berau River delta (Kalimantan, Indonesia), Geochim. Cosmochim. Ac., 186, 13-31, 2016.

Stoll, H. M., Moreno, A., Mendez-Vicente, A., Gonzalez-Lemos, S., Jimenez-Sanchez, M., Jose Dominguez-Cuesta, M., Edwards, R. L., Cheng, H., and Wang, X.: Paleoclimate and growth rates of speleothems in the northwestern Iberian Peninsula over the last two glacial cycles, Quaternary Res., 80, 284-290, 2013.

Stuiver, M., Reimer, P., and Braziunas, T.: High-precision radiocarbon age calibration for terrestrial and marine samples, Radiocarbon, 40, 1127-1151, 1998.

Trigo, R. M., Pozo-Vazquez, D., Osborn, T. J., Castro-Diez, Y., Gamiz-Fortis, S., and Esteban-Parra, M. J.: North Atlantic oscillation influence on precipitation, river flow and water resources in the Iberian peninsula, Int. J. Climatol., 24, 925-944, 2004.

Vale, C. and Catarino, F.: Accumulation of $\mathrm{Zn}, \mathrm{Pb}, \mathrm{Cu}, \mathrm{Cr}$ and $\mathrm{Ni}$ in sediments between roots of the Tagus estuary salt marshes, Portugal, Estuar. Coast. Shelf Res., 42, 393-403, 1996.

Vaz, N., Mateus, M., and Dias, J. M.: Semidiurnal and spring-neap variations in the Tagus Estuary: Application of a process-oriented hydro-biogeochemical model, J. Coastal. Res., 64, 1619-1623, 2011.

Vis, G.-J. and Kasse, C.: Late Quaternary valley-fill succession of the Lower Tagus Valley, Portugal, Sediment. Geol., 221, 19-39, 2009.

Vis, G.-J., Kasse, C., and Vandenberghe, J.: Late Pleistocene and Holocene palaeogeography of the Lower Tagus Valley (Portugal): effects of relative sea level, valley morphology and sediment supply, Quaternary Sci. Rev., 27, 1682-1709, 2008.

Vis, G.-J., Bohncke, S. J. P., Schneider, H., Kasse, C., CoenraadsNederveen, S., Zuurbier, K., and Rozema, J.: Holocene flooding history of the Lower Tagus Valley (Portugal), J. Quaternary Sci., 25, 1222-1238, 2010.

Vis, G.-J., Kasse, C., Kroon, D., Vandenberghe, J., Jung, S., Lebreiro, S. M., and Rodrigues, T.: Time-integrated 3D approach 
of late Quaternary sediment-depocenter migration in the Tagus depositional system: From river valley to abyssal plain, EarthSci. Rev., 153, 192-211, 2016.

Weber, Y., De Jonge, C., Rijpstra, W. I. C., Hopmans, E. C., Stadnitskaia, A., Schubert, C. J., Lehmann, M. F., Sinninghe Damsté, J. S., and Niemann, H.: Identification and carbon isotope composition of a novel branched GDGT isomer in lake sediments: Evidence for lacustrine branched GDGT production, Geochim. Cosmochim. Ac., 154, 118-129, 2015.

Weijers, J. W., Schouten, S., Hopmans, E. C., Geenevasen, J. A., David, O. R., Coleman, J. M., Pancost, R. D., and Sinninghe Damsté, J. S.: Membrane lipids of mesophilic anaerobic bacteria thriving in peats have typical archaeal traits, Environ. Microbiol., 8, 648-657, 2006.

Weijers, J. W., Schouten, S., van den Donker, J. C., Hopmans, E. C., and Sinninghe Damsté, J. S.: Environmental controls on bacterial tetraether membrane lipid distribution in soils, Geochim. Cosmochim. Ac., 71, 703-713, 2007a.

Weijers, J. W., Schefuß, E., Schouten, S., and Sinninghe Damsté, J. S.: Coupled thermal and hydrological evolution of tropical Africa over the last deglaciation, Science, 315, 1701-1704, 2007b.

Weijers, J. W. H., Wiesenberg, G. L. B., Bol, R., Hopmans, E. C., and Pancost, R. D.: Carbon isotopic composition of branched tetraether membrane lipids in soils suggest a rapid turnover and a heterotrophic life style of their source organism(s), Biogeosciences, 7, 2959-2973, doi:10.5194/bg-7-2959-2010, 2010.

Weijers, J. W. H., Bernhardt, B., Peterse, F., Werne, J. P., Dungait, J. A. J., Schouten, S., and Sinninghe Damsté, J. S.: Absence of seasonal patterns in MBT-CBT indices in mid-latitude soils, Geochim. Cosmochim. Ac., 75, 3179-3190, 2011.
Yang, G., Zhang, C. L., Xie, S., Chen, Z., Gao, M., Ge, Z., and Yang, Z.: Microbial glycerol dialkyl glycerol tetraethers from river water and soil near the Three Gorges Dam on the Yangtze River, Org. Geochem., 56, 40-50, 2013.

Zell, C., Kim, J.-H., Moreira-Turcq, P., Abril, G., Hopmans, E. C., Bonnet, M.-P., Sobrinho, R. L., and Sinninghe Damsté, J. S.: Disentangling the origins of branched tetraether lipids and crenarchaeol in the lower Amazon River: Implications for GDGT-based proxies, Limnol. Oceanogr., 58, 343-353, 2013.

Zell, C., Kim, J.-H., Balsinha, M., Dorhout, D., Fernandes, C., Baas, M., and Sinninghe Damsté, J. S.: Transport of branched tetraether lipids from the Tagus River basin to the coastal ocean of the Portuguese margin: consequences for the interpretation of the MBT'/CBT paleothermometer, Biogeosciences, 11, 5637-5655, doi:10.5194/bg-11-5637-2014, 2014.

Zell, C., Kim, J.-H., Dorhout, D., Baas, M., and Sinninghe Damsté, J. S.: Sources and distributions of branched tetraether lipids and crenarchaeol along the Portuguese continental margin: Implications for the BIT index, Cont. Shelf Res., 96, 34-44, 2015.

Zorita, E., Kharin, V., and Vonstorch, H.: The Atmospheric Circulation and Sea-Surface Temperature in the North-Atlantic Area in Winter - Their Interaction and Relevance for Iberian Precipitation, J. Climatol., 5, 1097-1108, 1992. 